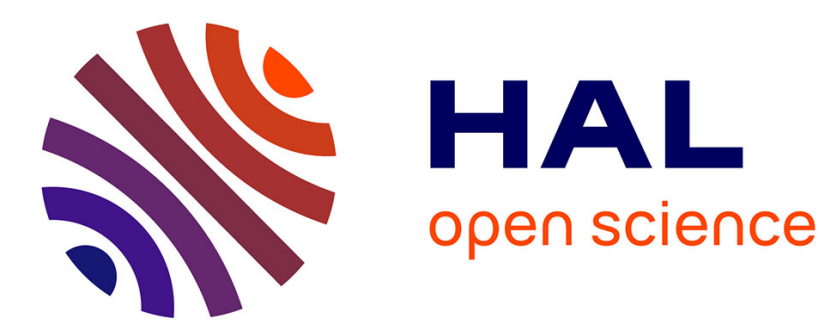

\title{
ON SUSPENDED BARITE AND TIIE OXYGEN MINIMUM IN THE SOUTHERN OCEAN
}

Frank Dehairs, L. Goeyens, N. Stroobants, P. Bernard, Catherine Goyet, Alain Poisson, R. Chesselet

\section{- To cite this version:}

Frank Dehairs, L. Goeyens, N. Stroobants, P. Bernard, Catherine Goyet, et al.. ON SUSPENDED BARITE AND TIIE OXYGEN MINIMUM IN THE SOUTHERN OCEAN. Global Biogeochemical Cycles, 1990, 4 (1), pp.85-102. 10.1029/GB004i001p00085 . hal-03561342

\author{
HAL Id: hal-03561342 \\ https://hal.science/hal-03561342
}

Submitted on 8 Feb 2022

HAL is a multi-disciplinary open access archive for the deposit and dissemination of scientific research documents, whether they are published or not. The documents may come from teaching and research institutions in France or abroad, or from public or private research centers.
L'archive ouverte pluridisciplinaire HAL, est destinée au dépôt et à la diffusion de documents scientifiques de niveau recherche, publiés ou non, émanant des établissements d'enseignement et de recherche français ou étrangers, des laboratoires publics ou privés. 


\section{ON SUSPENDED BARITE AND THE OXYGEN MINIMUM IN THE SOUTHERN OCEAN}

\author{
F. Dehairs, ${ }^{1}$ L. Goeyens, ${ }^{1}$ N. Stroobants, ${ }^{1}$ \\ P. Bernard, ${ }^{2}$ C. Goyet, ${ }^{3}$ A. Poisson, ${ }^{3}$ and \\ R. Chesselet ${ }^{4}$
}

Oxidation of locally produced organic matter, with which barite crystals are associated, consumes oxygen and sets free individual discrete barites. As a result oxygen decreases and barite increases away from the divergence, with barite integrating former biological processes.

\section{INTRODUCTION}

The important role of biological activity in the barium cycle was understood in earlier sedimentological studies, from the observed coincidence between high barite content in the sediments and presence of high plankton productivities in the overlying surface waters [Goldberg and Arthenius, 1958; Arrhenius and Bonatti, 1965; Church, 1970, 1976; Hanor, 1972].

The importance of biological control was confirmed from data on suspended matter collected during the Geochemical Ocean Sections (GEOSECS) program (1972-1979) showing barium in suspended matter to occur mainly as barite microcrystals and barite content to be positively correlated with plankton activity [Dehairs et al., 1980]. This is confirmed by Bishop [1989], who states that $\mathrm{Ba}$ appears to be a good indicator of ocean regions with intense organic production. Furthermore, data for sediment traps [Dymond, 1986] and deep-sea sediments [Schmitz, 1987] indicate the particulate barium-barite flux to be a proxy of the organic carbon flux. These latter studies show that despite the undersaturation of the water column for $\mathrm{BaSO}_{4}$ [Church, 1970, 1976; Church and Wolgemuth, 1972], barite behaves semiconservatively with respect to organic matter. However, its slow dissolution rate in the undersaturated water column was estimated to be sufficient to account for the main part of the dissolved barium input to the deep ocean [Dehairs et al., 1980; Rhein and Schlitzer, 1989].

While the general picture of biological control is now well established, details of the mechanisms of this 
control are lacking. Active intracellular production of barite is known to occur for some marine and freshwater algal and protozoan species [Hubert et al.,1975; Gayral and Fresnel, 1979; Brook et al., 1980; Finlay et al., 1983]. Chow and Goldberg [1960] proposed that formation of barite could also take place in supersaturated microenvironments composed of biogenic detritus. Evidence for barite association with microenvironments was indeed found during GEOSECS [Dehairs, 1979]. Furthermore, the use of high volume in-situ filuration devices clearly showed, for surface waters of the NW and SE Atlantic Ocean, the presence of barite in large aggregates of biogenic detritus enriched with diatom tests [Bishop, 1988]. Bishop also shows that below the surface waters barite is seen to shift from a large size fraction $(>53 \mu \mathrm{m})$ to a smaller size fraction $(<53 \mu \mathrm{m})$, probably as a result of aggregate breakdown. This process explains the subsurface particulate $\mathrm{Ba}$ maximum generally observed for profiles obtained using small volume, Niskin-type, samplers [Dehairs et al., 1980].

The aim of our research is to investigate further on the process of barite formation in the water column and on the usefulness of barite as a tracer of productivity. We specifically investigate on the mode of barite production (passive and/or active) as well as on the extent the subsurface Ba maximum, decribed above, reflects organic matter breakdown and oxidation. In the present study we compare the particulate $\mathrm{Ba}$ and dissolved oxygen data set we obtained for the Indien Geophysique Océan 3 (INDIGO 3) cruise in the southem ocean (Indian Ocean sector, January-February, 1987; R/V Marion Dufresne), during which marked oxygen minima and barium maxima were observed in subsurface waters. During INDIGO 3 we also investigated the possible impact of heterotrophic activity on barite formation by comparing rates of organic matter mineralization with occurrence of barite in suspended matter. Prior to this study, only a small data set on particulate barium, obtained during the GEOSECS Atlantic program, existed for the southern ocean [Dehairs, 1979].

\section{EXPERIMENT}

\section{The Sampling and Experiments at Sea}

The area investigated is shown in Figure 1. The positions of the main frontal systems are indicated, as deduced from the surface water temperature profile [Goffart and Hecq, 1988]. Their latitudinal positions are close to those given by Lutjeharms et al. [1983] for the same general area.

Suspended matter samples were taken using 30-L Niskin bottles mounted on a hydrowire. Casts generally went down to $2000 \mathrm{~m}$, but for some stations the whole water column was sampled. The Niskin bottles used were slightly modified to locate the outlet in the lower lid so as to ensure collecting rapidly settling large particles on the filter membranes during filtration. Suspended matter was collected on cellulose-ester membranes (MFMillipore AAWP: $\varnothing, 47 \mathrm{~mm}$; porosity, $0.8 \mu \mathrm{m}$ ), by applying pressure ( 0.3 bar) of filtered air at the top outlet of the bottles. In this way, approximately 3.5 hours were necessary to filter a $30-\mathrm{L}$ sample. After filtration the filters were rinsed with $20 \mathrm{~mL}$ deionized water adjusted to $\mathrm{pH} 7$, for carbonate particle preservation, and dried at $50^{\circ} \mathrm{C}$. Samples were stored in polycarbonate petri dishes at ambient temperature. Several blank filters were treated similarly. Samples were analyzed a few months later in the shore-based laboratory in Brussels.

It is important to stress here the fact that the type of sampler used allows for a representative sampling of the small and slowly settling particles, constituting the bulk of the suspended matter mass. Fast settling large particles, such as fecal pellets and aggregates, are not sampled in a statistically significant way by this sampling technique, as indicated by Bishop and Edmond [1976]. Such particles represent only a small fraction of the total suspended matter mass but carry most of the vertical flux. However, within the euphotic layer the large particle fraction can represent up to $50 \%$ of the total particle mass [Bishop and Edmond, 1976]. Their quantitative sampling requires the use of high-volume (several cubic meters) sarnpling and filtration devices as used by Bishop [1988].

Remineralization rates were determined using isotope dilution experiments. One liter water samples were spiked with labelled $\left({ }^{15} \mathrm{~N}\right)$ ammonium and incubated in glass bottles at the temperature of surface seawater for 24 hours or more [Goeyens and Dehairs, 1989]. The mass balance equations for $\mathrm{N}-\mathrm{NH}_{4}$ and for ${ }^{15} \mathrm{~N}$, used to estimate regeneration as well as incorporation of ammonium, are those described by Harrison [1983].

\section{Measurements on Board the Ship}

Salinity was measured on the practical salinity scale using a Guildline salinometer. Dissolved oxygen was measured by Winkler titration. Nitrate and ammonium were analyzed with a Technicon autoanalyzer AA II using the method described by Armstrong et al. [1967] and Grasshoff [1969].

\section{Measurements in the Shore-Based Laboratory in Brussels}

For complete dissolution of the barite an alkaline fusion technique derived from the one described by Burman et al. [1978] is used. Before the fusion process is performed, the organic filter substrate is oxidized by adding concentrated $\mathrm{HNO}_{3}$ and $\mathrm{H}_{2} \mathrm{O}_{2}(1$ and $3 \mathrm{~mL}$ respectively; all Merck Suprapur) to the filters in platinum crucibles and by heating until complete dessication. The fusion itself is carried out with a $\mathrm{LiBO}_{2}$ flux (50 mg per filter, Johnson \& Matthey, Specpure) at $1100^{\circ} \mathrm{C}$ for 1 hour. The formed glassy pearl is then dissolved in $\mathrm{HNO}_{3}$ under heating. The solution is brought to $10 \mathrm{~mL}$ with deionized water and is $5 \%$ in $\mathrm{LiBO}_{2}$ and $4 \%$ in $\mathrm{HNO}_{3}$. The determination of barium is carried out by inductively coupled plasma - optical emission spectrometry (ICP-OES), using a Jobin-Yvon 48 simultaneous spectrometer. The spectrometer is equiped with a demountable torch (Durr / Jobin-Yvon). The sample is introduced using a concentric pneumatic glass nebulizer (Meinhard type $\mathrm{C}$ ). The nebulizer argon flow is previously wetted with deionized water, to avoid clogging by salt crystalisation in the nebulizer tip. 


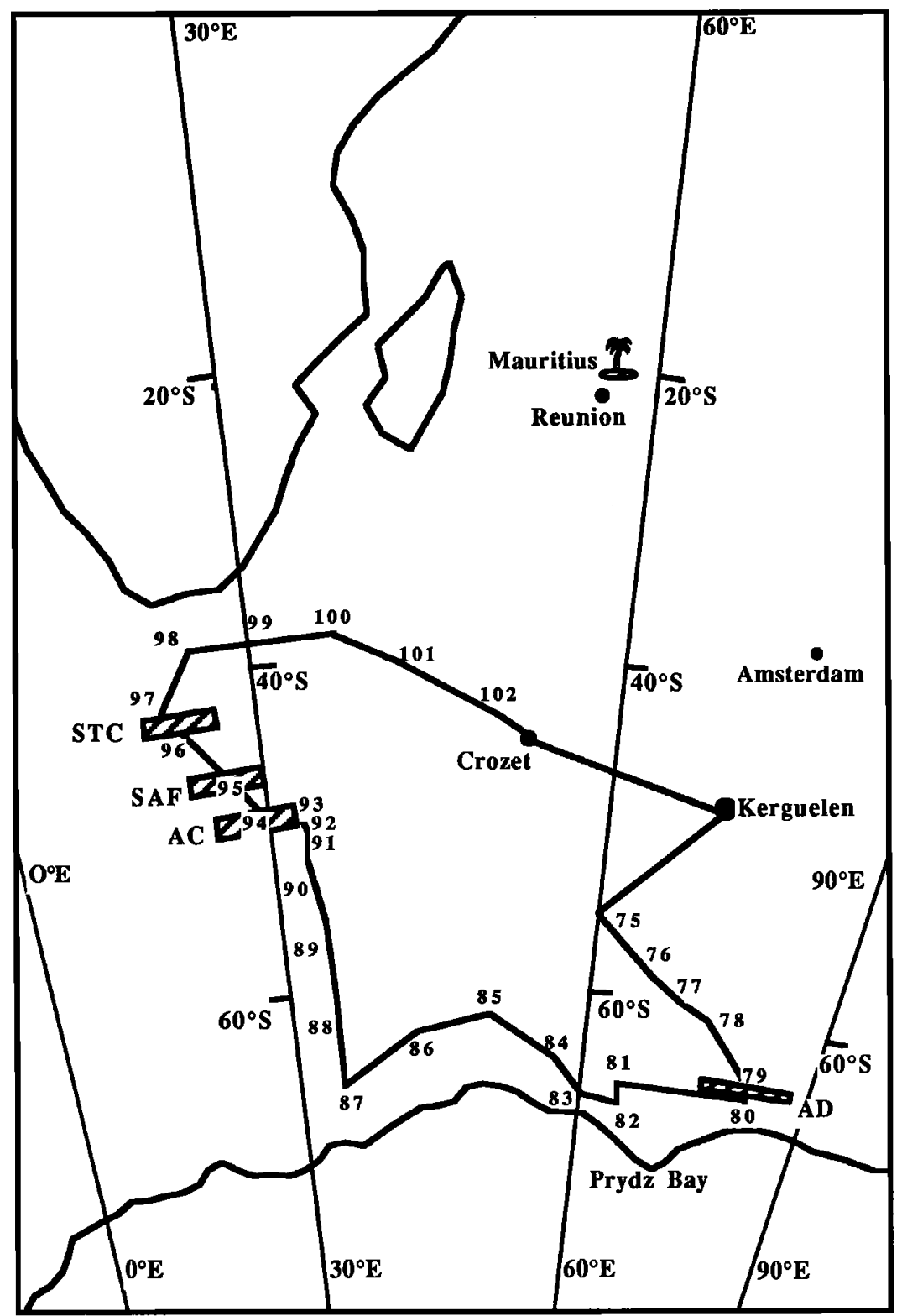

Fig. 1. INDIGO 3 cruise track in the Indian sector of the southern ocean. STC, Subtropical Convergence; SAF, Subantarctic Front; AC, Antarctic Convergence, or Polar Front; AD, Antarctic Divergence.

Operating power is generally $1.8 \mathrm{~kW}$. The argon flows are as follows: coolant, $19 \mathrm{~L} \mathrm{~min}^{-1}$; sample, $0.58 \mathrm{~L}$ min-1; auxiliary flow, $0.15 \mathrm{~L} \mathrm{~min}^{-1}$.

Barium standards (Titrisol, Merck) are prepared in the same matrix as the samples. Precision of the ICP determination (five consecutive measurements on the same solution) is between 1 and $5 \%$ for final solutions containing between 10 and $50 \mu \mathrm{g} \mathrm{L}^{-1}$ barium and better than $1.5 \%$ for solutions exceeding $50 \mathrm{\mu g} \mathrm{L}^{-1}$. Recovery of barium was checked using blank filter membranes spiked with $2 \mu \mathrm{g}$ barium. This showed a recovery of
$100 \%$. Procedural blanks were observed to contain $33 \pm$ $15 \mathrm{ng}$ Ba per filter $(\mathrm{n}=5)$.

Abundances of ${ }^{15} \mathrm{~N}$ are determined by emission spectrometric analysis with a Jasco, Model NIA-1, ${ }^{15} \mathrm{~N}$ analyzer. Ammonium is separated from its seawater matrix by addition of excess hydroxide and trapped in acid before conversion into molecular nitrogen by the modified Dumas method [L.Goeyens et al., manuscript in preparation, 1990]. The measurement of the ${ }^{15} \mathrm{~N}$ abundances is calibrated with certified standards (Hikari, Kogyo Co., Ltd). 


\section{RESULTS}

The total data set for suspended matter profiles of INDIGO 3 is given elsewhere [Dehairs and Goeyens, 1989; Poisson et al., 1990]. The barium data for 10 typical stations are shown in Table 1 together with data for potential temperature, salinity, $\sigma_{\theta}$, dissolved oxygen, calculated $\mathrm{pCO}_{2}$ (from measured total alkalinity and total inorganic carbon), and chlorophyll- $a$ (Chl- $a$ data, from Goffart and Hecq [1988]).
For one station (76) we checked if the total barium measured by ICP was indeed carried mainly by barite, as observed earlier [Dehairs et al., 1980]. To that purpose, known additional fractions (about $10 \mathrm{~L}$ ) of the seawater volume sampled at 500 and $700 \mathrm{~m}$ (i.e., oxygen minimum and below oxygen minimum) were filtered on Nuclepore membranes $(0.4 \mu \mathrm{m}$ pore size; $\emptyset 47 \mathrm{~mm})$ for scanning electron microscope - electron microprobe (SEM-EMP) investigations. These were carried out with a JEOL JXA 733 Superprobe and Tracor Northern

TABLE 1. Profiles of Salinity, Potential Temperature, Density, Dissolved Oxygen, Particulate Barium and Partial Pressure of $\mathrm{CO}_{2}$ for Stations 75, 76, 78, 81, 86, 87, 88, 89, 90, 95, 98, and 99

\begin{tabular}{|c|c|c|c|c|c|c|}
\hline $\begin{array}{l}\text { Depth, } \\
\text { m }\end{array}$ & $\begin{array}{l}\text { Salinity, } \\
\text { ppt }\end{array}$ & $\begin{array}{l}\text { Tpot, } \\
{ }^{\circ} \mathrm{C}\end{array}$ & $\sigma_{\theta}$ & $\begin{array}{c}\mathrm{O}_{2} \\
\mu \mathrm{mol} \mathrm{kg-1}\end{array}$ & $\begin{array}{c}\mathrm{Ba}, \\
\text { pmol kg-1 }\end{array}$ & $\begin{array}{c}\mathrm{pCO}_{2}, \\
\text { Hatm }\end{array}$ \\
\hline $\begin{array}{r}-10 \\
-20 \\
-35 \\
-50 \\
-75 \\
-100 \\
-200 \\
-300 \\
-400 \\
-500 \\
-600 \\
-700 \\
-800 \\
-900 \\
-1000 \\
-1100 \\
-1300 \\
-1500\end{array}$ & $\begin{array}{l}\text { Sta } \\
33.922 \\
33.922 \\
33.922 \\
33.924 \\
33.934 \\
33.958 \\
34.105 \\
34.436 \\
34.540 \\
34.604 \\
34.653 \\
34.681 \\
34.702 \\
34.714 \\
34.726 \\
34.738 \\
34.749 \\
34.750\end{array}$ & $\begin{array}{c}75: 569 \\
2.326 \\
2.316 \\
2.319 \\
2.311 \\
1.678 \\
0.945 \\
0.860 \\
1.910 \\
2.020 \\
2.066 \\
2.078 \\
2.051 \\
1.976 \\
1.901 \\
1.832 \\
1.777 \\
1.633 \\
1.524\end{array}$ & $\begin{array}{l}9^{09^{\prime} E ; W} \\
27.084 \\
27.084 \\
27.084 \\
27.086 \\
27.143 \\
27.212 \\
27.335 \\
27.528 \\
27.603 \\
27.651 \\
27.689 \\
27.714 \\
27.736 \\
27.752 \\
27.767 \\
27.781 \\
27.800 \\
27.817\end{array}$ & $\begin{array}{c}\text { Column, } 47 \\
330 \\
330 \\
330 \\
331 \\
334 \\
336 \\
289 \\
190 \\
176 \\
175 \\
177 \\
180 \\
183 \\
186 \\
189 \\
193 \\
198 \\
202\end{array}$ & $\begin{array}{r}7 m \\
110 \\
177 \\
174 \\
191 \\
81 \\
84 \\
398 \\
656 \\
654 \\
401 \\
340 \\
329 \\
237 \\
303 \\
322 \\
259 \\
174 \\
198\end{array}$ & $\begin{array}{r}325 \\
340 \\
351 \\
347 \\
345 \\
345 \\
413 \\
557 \\
577 \\
563 \\
548 \\
524 \\
525 \\
512 \\
525 \\
520 \\
490 \\
-\end{array}$ \\
\hline $\begin{array}{r}-10 \\
-41 \\
-72 \\
-92 \\
-123 \\
-153 \\
-179 \\
-204 \\
-256 \\
-307 \\
-382 \\
-478 \\
-573 \\
-669 \\
-764 \\
-860 \\
-955 \\
-1098 \\
-1242 \\
-1433 \\
-1591 \\
-1778 \\
-1919 \\
-2246\end{array}$ & $\begin{array}{l}{ }^{S t}{ }^{S} .665 \\
33.67 \\
34.691 \\
33.793 \\
33.87 \\
33.974 \\
34.119 \\
34.254 \\
34.39 \\
34.454 \\
34.561 \\
34.618 \\
34.654 \\
34.682 \\
34.704 \\
34.724 \\
34.735 \\
34.742 \\
34.748 \\
34.737 \\
34.745 \\
34.732 \\
34.725 \\
34.707\end{array}$ & $\begin{array}{c}n 76: 59 \circ \\
1.344 \\
1.225 \\
-0.267 \\
-1.226 \\
-1.401 \\
-0.712 \\
-0.076 \\
0.587 \\
1.57 \\
1.788 \\
1.926 \\
1.988 \\
2.003 \\
1.993 \\
1.964 \\
1.916 \\
1.858 \\
1.743 \\
1.603 \\
1.425 \\
1.223 \\
1.012 \\
0.873 \\
0.594\end{array}$ & $\begin{array}{l}9^{9} 55^{\prime} E ; V \\
26.956 \\
26.963 \\
27.075 \\
27.154 \\
27.244 \\
27.289 \\
27.35 \\
27.413 \\
27.512 \\
27.564 \\
27.707 \\
27.662 \\
27.701 \\
27.721 \\
27.746 \\
27.76 \\
27.772 \\
27.788 \\
27.801 \\
27.812 \\
27.82 \\
27.825 \\
27.827 \\
27.836\end{array}$ & $\begin{array}{c}\text { Column, } 4 \\
342 \\
343 \\
349 \\
350 \\
343 \\
315 \\
288 \\
261 \\
207 \\
187 \\
177 \\
175 \\
177 \\
181 \\
185 \\
189 \\
193 \\
197 \\
202 \\
203 \\
204 \\
206 \\
206 \\
208\end{array}$ & $\begin{array}{r}10 \\
151 \\
408 \\
145 \\
66 \\
48 \\
112 \\
201 \\
271 \\
408 \\
407 \\
541 \\
341 \\
395 \\
237 \\
290 \\
247 \\
219 \\
175 \\
169 \\
305 \\
133 \\
139 \\
99 \\
76\end{array}$ & $\begin{array}{r}318 \\
330 \\
32 \overline{-} \\
330 \\
- \\
448 \\
546 \\
571 \\
591 \\
566 \\
541 \\
525 \\
507 \\
481 \\
461 \\
469 \\
46 \overline{-} \\
46 \overline{-} \\
473\end{array}$ \\
\hline
\end{tabular}


TABLE 1. (continued)

\begin{tabular}{|c|c|c|c|c|c|c|}
\hline $\begin{array}{c}\text { Depth, } \\
\text { m }\end{array}$ & $\begin{array}{c}\text { Salinity, } \\
\text { ppt }\end{array}$ & $\begin{array}{c}\text { Tpot, } \\
{ }^{\circ}\end{array}$ & $\sigma_{\theta}$ & $\begin{array}{c}\mathrm{O}_{2} \\
\mu \mathrm{mol} \mathrm{kg}\end{array}$ & $\begin{array}{c}\mathrm{Ba}, \\
\text { pmol kg-1 }\end{array}$ & $\underset{\mu \mathrm{atm}}{\mathrm{pCO}_{2}}$ \\
\hline \multicolumn{7}{|c|}{ Station 76 (continued) } \\
\hline $\begin{array}{l}-2527 \\
-2808 \\
-3089 \\
-3744 \\
-4025\end{array}$ & $\begin{array}{l}34.556 \\
34.699 \\
34.685 \\
\mathbf{3 4 . 6 8 1} \\
\mathbf{3 4 . 6 6 1}\end{array}$ & $\begin{array}{l}0.377 \\
0.199 \\
0.035 \\
- \\
-\end{array}$ & $\begin{array}{l}27.84 \\
27.84 \\
27.847 \\
27.85 \\
27.857\end{array}$ & $\begin{array}{l}210 \\
214 \\
217 \\
229 \\
235\end{array}$ & $\begin{array}{l}425 \\
115 \\
160 \\
105 \\
160\end{array}$ & $\begin{array}{r}476 \\
478 \\
- \\
476 \\
467\end{array}$ \\
\hline $\begin{array}{r}-10 \\
-20 \\
-35 \\
-75 \\
-100 \\
-200 \\
-300 \\
-400 \\
-500 \\
-600 \\
-700 \\
-800 \\
-900 \\
-1100 \\
-1300 \\
-1500 \\
-1750 \\
-2000\end{array}$ & $\begin{array}{r}S t \\
33.699 \\
33.701 \\
33.805 \\
33.039 \\
34.312 \\
34.576 \\
34.634 \\
34.687 \\
34.699 \\
34.716 \\
34.728 \\
34.734 \\
34.737 \\
34.739 \\
34.514 \\
34.731 \\
34.719 \\
34.703\end{array}$ & $\begin{array}{c}78: 61^{\circ} 4 \\
1.447 \\
1.442 \\
1.389 \\
-1.542 \\
-0.213 \\
1.77 \\
1.882 \\
1.885 \\
1.861 \\
1.816 \\
1.735 \\
1.681 \\
1.574 \\
1.398 \\
1.203 \\
1.04 \\
0.806 \\
0.602\end{array}$ & $\begin{array}{c}6^{\circ} 16^{\circ} E ; \\
26.963 \\
26.962 \\
26.97 \\
27.381 \\
27.496 \\
27.646 \\
27.699 \\
27.726 \\
27.743 \\
27.758 \\
27.773 \\
27.785 \\
27.795 \\
27.809 \\
27.819 \\
27.83 \\
27.835 \\
27.831\end{array}$ & $\begin{array}{c}r \text { Column, } \\
349 \\
349 \\
348 \\
307 \\
251 \\
178 \\
174 \\
177 \\
181 \\
185 \\
192 \\
192 \\
195 \\
199 \\
203 \\
204 \\
205 \\
206\end{array}$ & $\begin{array}{r}51 m \\
1471 \\
1250 \\
2240 \\
149 \\
190 \\
569 \\
432 \\
342 \\
374 \\
- \\
278 \\
254 \\
224 \\
201 \\
324 \\
124 \\
130 \\
147\end{array}$ & $\begin{array}{r}282 \\
280 \\
- \\
387 \\
466 \\
602 \\
- \\
567 \\
538 \\
- \\
512 \\
493 \\
501 \\
491 \\
467 \\
487 \\
492 \\
485\end{array}$ \\
\hline
\end{tabular}

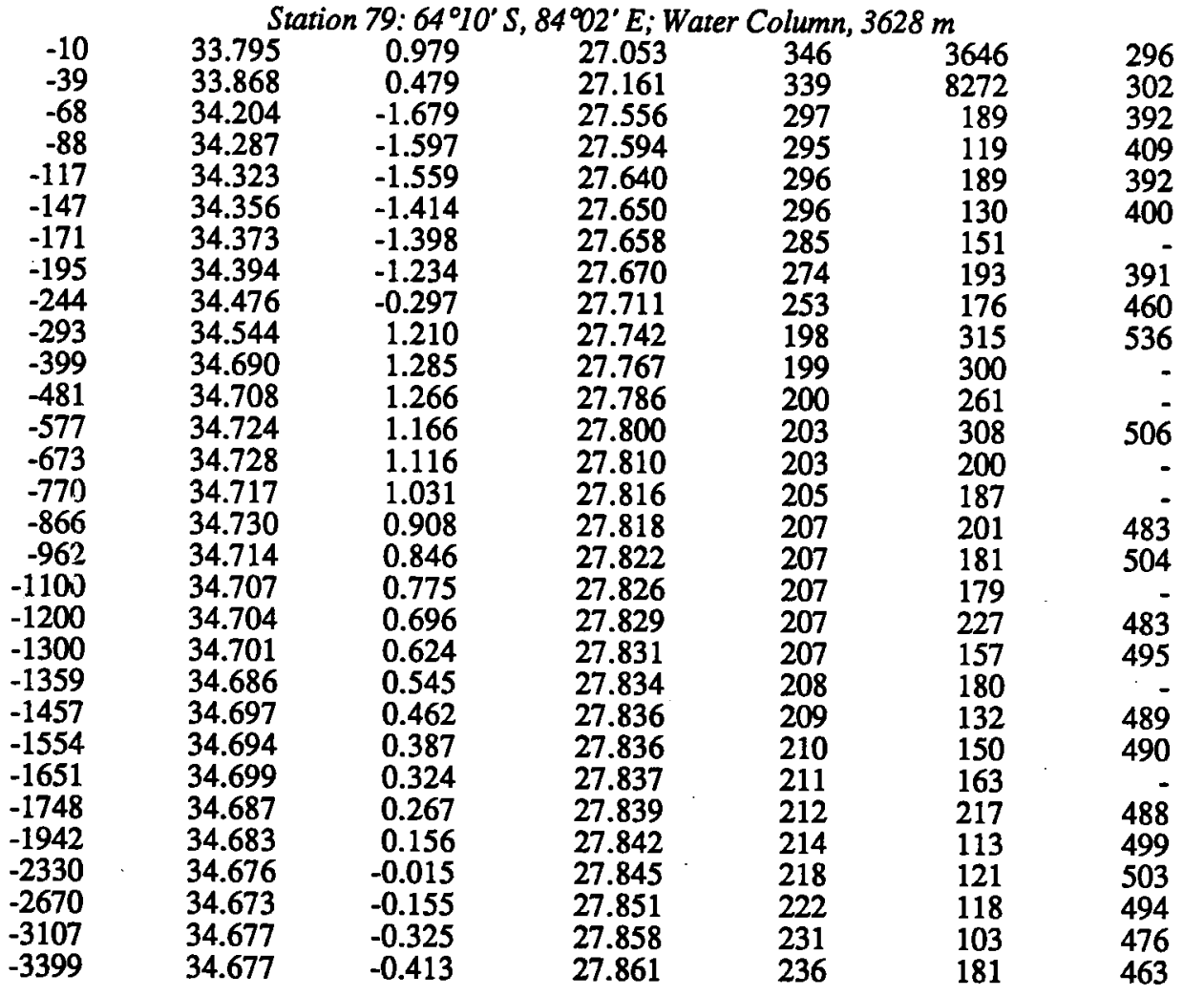


TABLE 1. (continued)

\begin{tabular}{|c|c|c|c|c|c|c|}
\hline $\begin{array}{l}\text { Depth, } \\
\text { m }\end{array}$ & $\begin{array}{c}\text { Salinity, } \\
\text { ppt }\end{array}$ & $\begin{array}{l}\text { Tpot, } \\
{ }^{\circ}\end{array}$ & $\sigma_{\theta}$ & $\begin{array}{c}\mathrm{O}_{2} \\
\mu \mathrm{mol} \mathrm{kg}^{-1}\end{array}$ & $\begin{array}{c}\mathrm{Ba}, \\
\text { pmol kg-1 }^{-1}\end{array}$ & $\underset{\text { Hatm }}{\mathrm{pCO}_{2},}$ \\
\hline \multicolumn{7}{|c|}{ Station 81: 65 ${ }^{\circ} 59^{\prime} \mathrm{S}, 67^{\circ} 16^{\prime} \mathrm{E} ;$ Water Column, $3098 \mathrm{~m}$} \\
\hline-10 & 34.143 & 0.295 & 27.382 & 340 & 114 & 358 \\
\hline-25 & 34.146 & 0.266 & 27.386 & 340 & 49 & 357 \\
\hline-50 & 34.320 & -1.442 & 27.618 & 313 & 91 & 392 \\
\hline-75 & 34.413 & -1.633 & 27.701 & 292 & 108 & 445 \\
\hline-100 & 34.428 & -1.646 & 27.715 & 290 & 110 & 370 \\
\hline-200 & 34.503 & -0.983 & 27.745 & 272 & 109 & 404 \\
\hline-300 & 34.620 & 0.227 & 27.787 & 232 & 248 & 452 \\
\hline-400 & 34.670 & 0.763 & 27.802 & 215 & 283 & 477 \\
\hline-500 & 34.678 & 0.715 & 27.822 & 214 & 280 & 484 \\
\hline-600 & 34.688 & 0.758 & 27.819 & 211 & 225 & 486 \\
\hline-700 & 34.688 & 0.660 & 27.819 & 212 & 236 & 476 \\
\hline-800 & 34.699 & 0.669 & 27.826 & 210 & 214 & 495 \\
\hline-900 & 34.697 & 0.619 & 27.822 & 209 & 178 & 488 \\
\hline-1000 & 34.697 & 0.550 & 27.827 & 209 & 181 & - \\
\hline-1100 & 34.698 & 0.483 & 27.831 & 209 & 215 & 496 \\
\hline-1300 & 34.690 & 0.363 & 27.841 & 210 & 145 & - \\
\hline-1500 & 34.688 & 0.221 & 27.845 & 213 & 176 & 469 \\
\hline-1750 & 34.687 & 0.091 & 27.844 & 216 & 171 & 455 \\
\hline-2000 & 34.687 & -0.018 & 27.847 & 219 & 135 & \\
\hline
\end{tabular}

Station 86: 63\%45' S, $41^{\circ 59} 9^{\prime} \mathrm{E}$; Water Column, $4688 \mathrm{~m}$

$\begin{array}{rllllrr}-10 & 33.858 & 0.821 & 27.136 & 339 & 32 & 347 \\ -25 & 33.854 & 0.81 & 27.137 & 339 & 113 & 367 \\ -35 & 33.864 & 0.74 & 27.147 & 338 & 195 & 359 \\ -50 & 33.873 & 0.644 & 27.343 & 332 & 164 & 346 \\ -75 & 34.195 & 1.607 & 27.51 & 318 & 31 & 344 \\ -100 & 34.335 & 0.123 & 27.597 & 253 & 155 & 472 \\ -200 & 34.645 & 1.441 & 27.727 & 186 & 543 & 569 \\ -300 & 34.684 & 1.421 & 27.764 & 189 & 207 & 557 \\ -400 & 34.705 & 1.401 & 27.785 & 193 & 361 & 532 \\ -500 & 34.714 & 1.3 & 27.798 & 196 & 341 & 526 \\ -600 & 34.721 & 1.195 & 27.799 & 198 & 216 & 517 \\ -700 & 34.723 & 1.076 & 27.811 & 201 & 186 & - \\ -800 & 34.719 & 0.959 & 27.819 & 203 & 171 & 5 \\ -900 & 34.713 & 0.846 & 27.822 & 204 & 292 & 513 \\ -1000 & 34.71 & 0.752 & 27.825 & 205 & 537 & - \\ -1100 & 34.705 & 0.658 & 27.829 & 206 & 246 & 494 \\ -1300 & 34.696 & 0.48 & 27.833 & 208 & 120 & 500 \\ -1500 & 34.693 & 0.344 & 27.836 & 209 & 100 & 494 \\ -1750 & 34.686 & 0.225 & 27.839 & 210 & 144 & 503 \\ -2000 & 34.683 & 0.106 & 27.844 & 215 & 130 & 494\end{array}$

\begin{tabular}{rllllrr}
\multicolumn{7}{c}{ Station $87: 65^{\circ} 0^{\prime} S, 32$ OI $E ;$ Water Column, $4584 m$} \\
-10 & 33.857 & 1.057 & 27.123 & 340 & 58 & 347 \\
-30 & 33.946 & 0.76 & 27.19 & 339 & 119 & - \\
-50 & 34.239 & -1.525 & 27.535 & 328 & 61 & 343 \\
-75 & 34.314 & -1.397 & 27.61 & 307 & 62 & 376 \\
-100 & 34.411 & -0.678 & 27.662 & 265 & 83 & 448 \\
-200 & 34.659 & 1.366 & 27.747 & 190 & 367 & 543 \\
-300 & 34.683 & 1.268 & 27.773 & 195 & 212 & 523 \\
-400 & 34.696 & 1.165 & 27.79 & 199 & 156 & 520 \\
-500 & 34.701 & 1.125 & 27.798 & 201 & 260 & 521 \\
-600 & 34.713 & 1.035 & 27.813 & 201 & 115 & 502 \\
-700 & 34.714 & 0.941 & 27.822 & 203 & 191 & 501 \\
-800 & 34.709 & 0.762 & 27.826 & 204 & 157 & - \\
-900 & 34.701 & 0.722 & 27.826 & 206 & 116 & 479 \\
-1000 & 34.698 & 0.592 & 27.829 & 207 & 147 & -
\end{tabular}


TABLE 1. (continued)

\begin{tabular}{|c|c|c|c|c|c|c|}
\hline $\begin{array}{c}\text { Depth, } \\
\text { m }\end{array}$ & $\begin{array}{c}\text { Salinity, } \\
\text { ppt }\end{array}$ & $\begin{array}{c}\text { Tpot, } \\
{ }^{C}\end{array}$ & $\sigma_{\theta}$ & $\begin{array}{c}\mathrm{O}_{2} \\
\mu \mathrm{mol} \mathrm{kg}\end{array}$ & $\begin{array}{c}\mathrm{Ba}, \\
\text { pmol kg }^{-1}\end{array}$ & 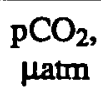 \\
\hline \multicolumn{7}{|c|}{ Station 87 (continued) } \\
\hline $\begin{array}{l}-1100 \\
-1300 \\
-1500 \\
-1750 \\
-2000\end{array}$ & $\begin{array}{l}34.695 \\
34.688 \\
34.679 \\
34.677 \\
34.673\end{array}$ & $\begin{array}{r}0.505 \\
0.347 \\
0.224 \\
0.079 \\
-0.063\end{array}$ & $\begin{array}{l}27.832 \\
27.836 \\
27.835 \\
27.841 \\
27.834\end{array}$ & $\begin{array}{l}208 \\
209 \\
213 \\
215 \\
219\end{array}$ & $\begin{array}{r}128 \\
146 \\
96 \\
146 \\
118\end{array}$ & $\begin{array}{l}494 \\
479 \\
473 \\
493 \\
487\end{array}$ \\
\hline $\begin{array}{r}-10 \\
-40 \\
-70 \\
-90 \\
-120 \\
-150 \\
-175 \\
-200 \\
-250 \\
-300 \\
-400 \\
-500 \\
-600 \\
-700 \\
-800 \\
-900 \\
-1000 \\
-1150 \\
-1300 \\
-1500 \\
-1700 \\
-1900 \\
-2150 \\
-2400 \\
-2700 \\
-3000 \\
-3300 \\
-3700 \\
-4100 \\
-4500\end{array}$ & $\begin{array}{l}\text { Sta: } \\
33.786 \\
33.788 \\
33.915 \\
34.013 \\
34.162 \\
34.313 \\
34.439 \\
34.565 \\
34.627 \\
34.650 \\
34.681 \\
34.706 \\
34.712 \\
34.714 \\
34.715 \\
34.711 \\
34.707 \\
34.701 \\
34.695 \\
34.688 \\
34.682 \\
34.678 \\
34.682 \\
34.680 \\
34.667 \\
34.666 \\
34.664 \\
34.662 \\
34.658 \\
34.655\end{array}$ & $\begin{array}{c}88: 610 \\
1.305 \\
1.119 \\
-1.042 \\
-1.425 \\
-1.019 \\
0.314 \\
0.794 \\
1.293 \\
1.431 \\
1.448 \\
1.425 \\
1.371 \\
1.281 \\
1.168 \\
1.046 \\
0.911 \\
0.802 \\
0.636 \\
0.494 \\
0.334 \\
0.224 \\
0.133 \\
0.013 \\
-0.095 \\
-0.206 \\
0.303 \\
-0.389 \\
0.488 \\
-0.570 \\
-0.639\end{array}$ & $\begin{array}{l}{ }^{\circ} 17^{\prime} E ; \boldsymbol{W} \\
27.049 \\
27.064 \\
27.319 \\
27.400 \\
27.473 \\
27.585 \\
27.630 \\
27.677 \\
27.717 \\
27.734 \\
27.761 \\
27.785 \\
27.796 \\
27.806 \\
27.814 \\
27.820 \\
27.824 \\
27.829 \\
27.833 \\
27.836 \\
27.838 \\
27.839 \\
27.849 \\
27.853 \\
27.849 \\
27.853 \\
27.855 \\
27.857 \\
27.857 \\
27.858\end{array}$ & $\begin{array}{c}\text { Column, } 51 \\
342 \\
342 \\
341 \\
338 \\
308 \\
243 \\
220 \\
196 \\
189 \\
188 \\
190 \\
193 \\
197 \\
199 \\
201 \\
203 \\
204 \\
206 \\
208 \\
209 \\
210 \\
214 \\
217 \\
220 \\
225 \\
229 \\
232 \\
237 \\
240 \\
242\end{array}$ & $\begin{array}{r}9 m \\
319 \\
384 \\
37 \\
85 \\
136 \\
233 \\
406 \\
535 \\
492 \\
337 \\
327 \\
261 \\
302 \\
263 \\
357 \\
276 \\
230 \\
230 \\
196 \\
222 \\
142 \\
<4 \\
46 \\
109 \\
67 \\
67 \\
81 \\
73 \\
83 \\
45\end{array}$ & $\begin{array}{r}315 \\
314 \\
342 \\
354 \\
397 \\
497 \\
- \\
557 \\
- \\
556 \\
561 \\
527 \\
529 \\
499 \\
- \\
509 \\
- \\
482 \\
496 \\
496 \\
509 \\
503 \\
471 \\
- \\
- \\
- \\
454 \\
446 \\
454\end{array}$ \\
\hline $\begin{array}{r}-10 \\
-30 \\
-50 \\
-75 \\
-100 \\
-200 \\
-300 \\
-400 \\
-500 \\
-600 \\
-700 \\
-800 \\
-900 \\
-1000 \\
-1100 \\
-1300 \\
-1500 \\
-1750 \\
-2000\end{array}$ & $\begin{array}{l}\quad \text { Sta } \\
33.939 \\
33.948 \\
33.965 \\
33.983 \\
34.026 \\
34.298 \\
34.506 \\
34.608 \\
34.672 \\
34.694 \\
34.72 \\
34.746 \\
34.73 \\
34.731 \\
34.737 \\
34.726 \\
34.722 \\
34.7 \\
34.701\end{array}$ & $\begin{array}{c}89: 566^{\circ} \\
2.847 \\
1.908 \\
1.867 \\
1.411 \\
-0.015 \\
1.136 \\
1.882 \\
1.763 \\
1.718 \\
1.689 \\
1.635 \\
1.541 \\
1.399 \\
1.299 \\
1.196 \\
0.975 \\
0.729 \\
0.497 \\
0.324\end{array}$ & $\begin{array}{c}500^{\prime} E ; W \\
27.055 \\
27.142 \\
27.156 \\
27.205 \\
27.328 \\
27.483 \\
27.617 \\
27.681 \\
27.72 \\
27.748 \\
27.766 \\
27.782 \\
27.796 \\
27.803 \\
27.811 \\
27.823 \\
27.827 \\
27.832 \\
27.838\end{array}$ & $\begin{array}{c}\text { Column, } 542 \\
326 \\
333 \\
335 \\
332 \\
332 \\
241 \\
180 \\
180 \\
181 \\
185 \\
189 \\
193 \\
196 \\
198 \\
200 \\
204 \\
206 \\
208 \\
210\end{array}$ & $\begin{array}{r}50 \\
93 \\
53 \\
135 \\
189 \\
185 \\
355 \\
398 \\
415 \\
291 \\
286 \\
295 \\
259 \\
194 \\
251 \\
224 \\
244 \\
177 \\
142 \\
157\end{array}$ & $\begin{array}{r}352 \\
337 \\
355 \\
353 \\
369 \\
516 \\
617 \\
584 \\
571 \\
546 \\
536 \\
- \\
505 \\
- \\
- \\
496 \\
505 \\
487 \\
483\end{array}$ \\
\hline
\end{tabular}




\begin{tabular}{rrrrrrr}
\multicolumn{7}{c}{ Station $90: 530^{\prime} I^{\prime} S, 31^{\circ}{ }^{\circ} 3^{\prime} E ;$ Water Column, $5260 m$} \\
-10 & 33.947 & 3.854 & 26.961 & 323 & 94 & 353 \\
-30 & 33.953 & 3.645 & 26.985 & 326 & 385 & 354 \\
-50 & 33.989 & 2.046 & 27.135 & 330 & 220 & 355 \\
-75 & 34.032 & 1.422 & 27.234 & 325 & 140 & 383 \\
-100 & 34.045 & 1.219 & 27.264 & 321 & 200 & 361 \\
-200 & 34.237 & 1.061 & 27.429 & 257 & 575 & 499 \\
-300 & 34.487 & 1.882 & 27.571 & 179 & 412 & 588 \\
-400 & 34.533 & 1.683 & 27.623 & 182 & 291 & 598 \\
-500 & 34.617 & 1.898 & 27.674 & 173 & 184 & 566 \\
-600 & 34.640 & 1.764 & 27.703 & 176 & 200 & 576 \\
-700 & 34.673 & 1.784 & 27.725 & 178 & 223 & - \\
-800 & 34.701 & 1.789 & 27.747 & 183 & 201 & - \\
-900 & 34.722 & 1.773 & 27.768 & 188 & 122 & 527 \\
-1000 & 34.731 & 1.700 & 27.779 & 192 & 202 & - \\
-1100 & 34.738 & 1.626 & 27.792 & 194 & 166 & 494 \\
-1300 & 34.738 & 1.431 & 27.806 & 198 & 131 & 486 \\
-1750 & 34.718 & 0.876 & 27.824 & 205 & 137 & 483 \\
-2000 & 34.708 & 0.577 & 27.832 & 208 & 127 & -
\end{tabular}

-10
-30
-50
-7
-100
-200
-300
-400
-500
-600
-700
-800
-900
-1000
-1100
-1300
-1500
-1750
-2000

$-10$

$$
-50
$$

$-100$

$-200$

$-300$

$-400$

$-500$

$-600$

$-700$

$-800$

$-900$

$-1000$

$-1100$

$-1300$

$-1500$

$-1750$

$-10$
Station 95: 47 ${ }^{\circ} 9^{\prime}$ ' S, $23^{\circ}$

\subsection{3}

$$
33.879
$$

33.884

33.897

33.951

34.095

34.186

34.183

34.205

34.264

34.332

34.98

34.46

34.516

34.571

34.659

34.712

34.786
34.765

\begin{abstract}
6.868
\end{abstract}
6.769

6.526

6.2

5.216

4.346

3.737

3.22

2.847

2.632

2.613

2.571

2.503

2.466

2.429

2.417

2.358

2.213

1.898

\subsection{1}

26.569

26.608

26.658

26.821

27.032

27.167

27.215

27.266

27.332

27.388

27.444

27.5

27.547

27.595

27.666

27.713

27.768

27.802
Column, $5559 \mathrm{~m}$

298 , $89 \quad 362$

$\begin{array}{lll}298 & 105 & 367\end{array}$

$\begin{array}{lll}300 & 76 & 363\end{array}$

$\begin{array}{lll}301 & 88 & 358\end{array}$

$303 \quad 95 \quad 371$

$286 \quad 176 \quad 403$

$267 \quad 206 \quad 452$

$260 \quad 209 \quad 440$

$247 \quad 279 \quad 464$

$226 \quad 249 \quad 557$

$209 \quad 180$

$185 \quad 220$

$185 \quad 153$

$182 \quad 139$

$179 \quad 111$

$\begin{array}{ll}180 & 167 \\ 186 & 148\end{array}$

$197 \quad 123$

$204 \quad 69$

$69 \quad 47 \overline{8}^{-}$

Station 98: $38^{\circ} 00^{\prime} S, 3^{\circ} 18^{\circ} \mathrm{E}$; Water Column, $5370 \mathrm{~m}$

$\begin{array}{llllrl}35.487 & 23.056 & 24.291 & 213 & 384 & 331 \\ 35.499 & 22.228 & 24.537 & 216 & 1174 & 330 \\ 35.539 & 21.808 & 24.685 & 212 & 571 & 332 \\ 35.566 & 21.256 & 24.858 & 209 & 268 & \\ 35.552 & 20.345 & 24.74 & 196 & 402 & 362 \\ 35.609 & 17.576 & 25.845 & 213 & 145 & 362 \\ 35.469 & 15.557 & 26.212 & 199 & 189 & 395 \\ 35.328 & 14.058 & 26.431 & 208 & 216 & 397 \\ 35.196 & 12.929 & 26.561 & 208 & 208 & 407 \\ 35.024 & 11.683 & 26.67 & 214 & 89 & 414 \\ 34.884 & 10.463 & 26.779 & 210 & 110 & 439 \\ 34.745 & 9.096 & 26.899 & 204 & 98 & \\ 34.607 & 7.57 & 27.031 & 196 & 129 & 503 \\ 34.527 & 6.487 & 27.111 & 197 & 368 & \\ 34.448 & 5.425 & 27.19 & 198 & 180 & 536 \\ 34.428 & 3.917 & 27.342 & 190 & 138 & 572 \\ 34.543 & 3.418 & 27.483 & 169 & 127 & 611 \\ 34.6 & 2.724 & 27.592 & 177 & 134 & 589\end{array}$

Station 99: 37059' S, 30॰7' E; Water Column, $4129 \mathrm{~m}$

$\begin{array}{llllll}35.682 & 21.711 & 24.821 & 219 & 59 & 330 \\ 35.68 & 21.721 & 24.819 & 220 & 23 & 324\end{array}$


Dehairs et al.: Suspended Barite and Southern Ocean Oxygen Minimum

TABLE 1. (continued)

\begin{tabular}{|c|c|c|c|c|c|c|}
\hline $\begin{array}{l}\text { Depth, } \\
\text { m }\end{array}$ & $\begin{array}{c}\text { Salinity, } \\
\text { ppt }\end{array}$ & $\begin{array}{c}\text { Tpot, } \\
{ }^{\circ} \mathrm{C}\end{array}$ & $\sigma_{\theta}$ & $\begin{array}{c}\mathrm{O}_{2} \\
\mu \mathrm{mol} \mathrm{kg}^{-1}\end{array}$ & $\begin{array}{c}\mathrm{Ba} \\
\text { pmol kg-1 }\end{array}$ & 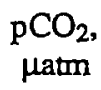 \\
\hline \multicolumn{7}{|c|}{ Station 99 (continued) } \\
\hline $\begin{array}{r}-50 \\
-75 \\
-100 \\
-200 \\
-300 \\
-400 \\
-500 \\
-600 \\
-700 \\
-800 \\
-900 \\
-1000 \\
-1100 \\
-1300 \\
-1500 \\
-1750\end{array}$ & $\begin{array}{l}35.681 \\
35.605 \\
35.615 \\
35.62 \\
35.615 \\
35.418 \\
35.234 \\
35.116 \\
34.978 \\
34.824 \\
34.719 \\
34.602 \\
34.501 \\
34.385 \\
34.493 \\
34.592\end{array}$ & $\begin{array}{l}21.714 \\
19.894 \\
18.914 \\
16.996 \\
16.835 \\
15.1 \\
13.521 \\
12.463 \\
11.298 \\
10.042 \\
8.835 \\
7.492 \\
6.371 \\
4.247 \\
3.627 \\
2.964\end{array}$ & $\begin{array}{l}24.822 \\
25.255 \\
25.516 \\
25.993 \\
26.028 \\
26.274 \\
26.47 \\
26.59 \\
26.702 \\
26.81 \\
26.918 \\
27.038 \\
27.113 \\
27.273 \\
27.423 \\
27.565\end{array}$ & $\begin{array}{l}220 \\
217 \\
209 \\
224 \\
226 \\
206 \\
216 \\
214 \\
212 \\
211 \\
203 \\
193 \\
198 \\
203 \\
178 \\
171\end{array}$ & $\begin{array}{r}37 \\
77 \\
92 \\
142 \\
92 \\
147 \\
126 \\
177 \\
128 \\
122 \\
114 \\
145 \\
89 \\
162 \\
165 \\
138\end{array}$ & $\begin{array}{r}326 \\
338 \\
347 \\
346 \\
337 \\
388 \\
389 \\
414 \\
- \\
445 \\
- \\
522 \\
530 \\
546 \\
538 \\
617\end{array}$ \\
\hline
\end{tabular}

energy dispersive $\mathrm{X}$-ray detector, using an automatic particle recognition program [Raeymakers, 1986; Storms, 1988]. Barite is observed to occur as discrete particles with log-normal size distributions, having their modes at 1 and $0.84 \mu \mathrm{m}$, respectively. Smallest crystals recorded are $0.32 \mu \mathrm{m}$ at $500 \mathrm{~m}$ and $0.08 \mu \mathrm{m}$ at $700 \mathrm{~m}$. The barium quantities carried by the detected barite crystals, normalized to unit volume, were compared with those obtained by ICP-OES. This comparative study shows that $81 \%$ (at $700 \mathrm{~m}$ ) to $86 \%$ (at $500 \mathrm{~m}$ ) of the total barium is carried by barite microcrystals, thus confirming earlier results [Dehairs et al., 1980]. This good agreement between barite and total barium is also consistent with the high barium to aluminum ratio we observe in total suspended matter (these $\mathrm{Al}$ data are reported by Poisson et al. [1990]) relative to crustal material. This indicates that excess barium is present which is not carried by the aluminosilicate fraction in the suspended matter.

\section{DISCUSSION}

For some of the stations given in Table 1, particulate barium profiles are compared with profiles of dissolved oxygen and $\mathrm{CO}_{2}$ partial pressure (Figure 2). From Table 1 and Figure 2, it appears that for the stations south of the Polar Front, barium maxima occur within the oxygen minimum or above it, in the gradient leading towards the oxygen minimum. However, some of these stations (78, 79,98 ) show also very high barium values in the euphotic layer, eventually exceeding the barium maximum associated with the oxygen minimum. Moreover, in some isolated cases, deepwater barium peaks do occur. These often coincide with increased concentrations of particulate $\mathrm{Ca}, \mathrm{Sr}$, and $\mathrm{Si}$ (data reported by Poisson et al. [1990]). We tentatively explain such deepwater maxima to result through the occasional disintegration of large, fast settling carrier particles en route to the sediments and which thereby inject their load of small particles into the deep water column.

Next we will focus the discussion on the occurrence of the barium maxima in the euphotic layer and in the oxygen minimum layer.

\section{The Barium Maximum in the Region of the Oxygen Minimum and $\mathrm{PCO}_{2}$ Maximum}

For all stations between the Polar Front and the Antarctic Divergence, the oxygen minimum and $\mathrm{pCO}_{2}$ maximum are located between 200 and $500 \mathrm{~m}$ depth. The barium maximum is located in the same depth interval, but peak values do not always coincide. Neither the oxygen minimum nor the barium maximum is located on isopycnal surfaces. In the oxygen minimum, $\sigma_{\theta}$ decreases from south to north (e.g., station $87,65^{\circ} 11^{\prime}$ S, $\sigma_{\theta}=27.747$; station $94,50^{\circ} 35^{\prime} \mathrm{S}, \sigma_{\theta}=27.657$ ). Oxygen saturation values in the oxygen minimum fit into a very narrow range (from 346 to $352 \mu \mathrm{M}$ ), while this range is wider for oxygen saturation values taken along isopycnal surfaces (e.g., $\sigma_{\theta}=27.700$; oxygen saturation between 347 and $371 \mu \mathrm{M})$. These observations suggest a common origin for the oxygen minimum waters and also suggest water movement, to some degree, to occur diapycnally.

The highest values for oxygen in the oxygen minimum and the lowest values for barium in the barium maximum are found at the southernmost stations. Between $65^{\circ} \mathrm{S}$ and $55^{\circ} \mathrm{S}$ oxygen decreases northwand, while barium and $\mathrm{pCO}_{2}$ decrease southward. In Figure 3 we plot barium, integrated between 200 and $700 \mathrm{~m}$ (that is the depth region in which the maximum is located) versus the dissolved oxygen content in the oxygen minimum for the stations located between the divergence and the Polar 

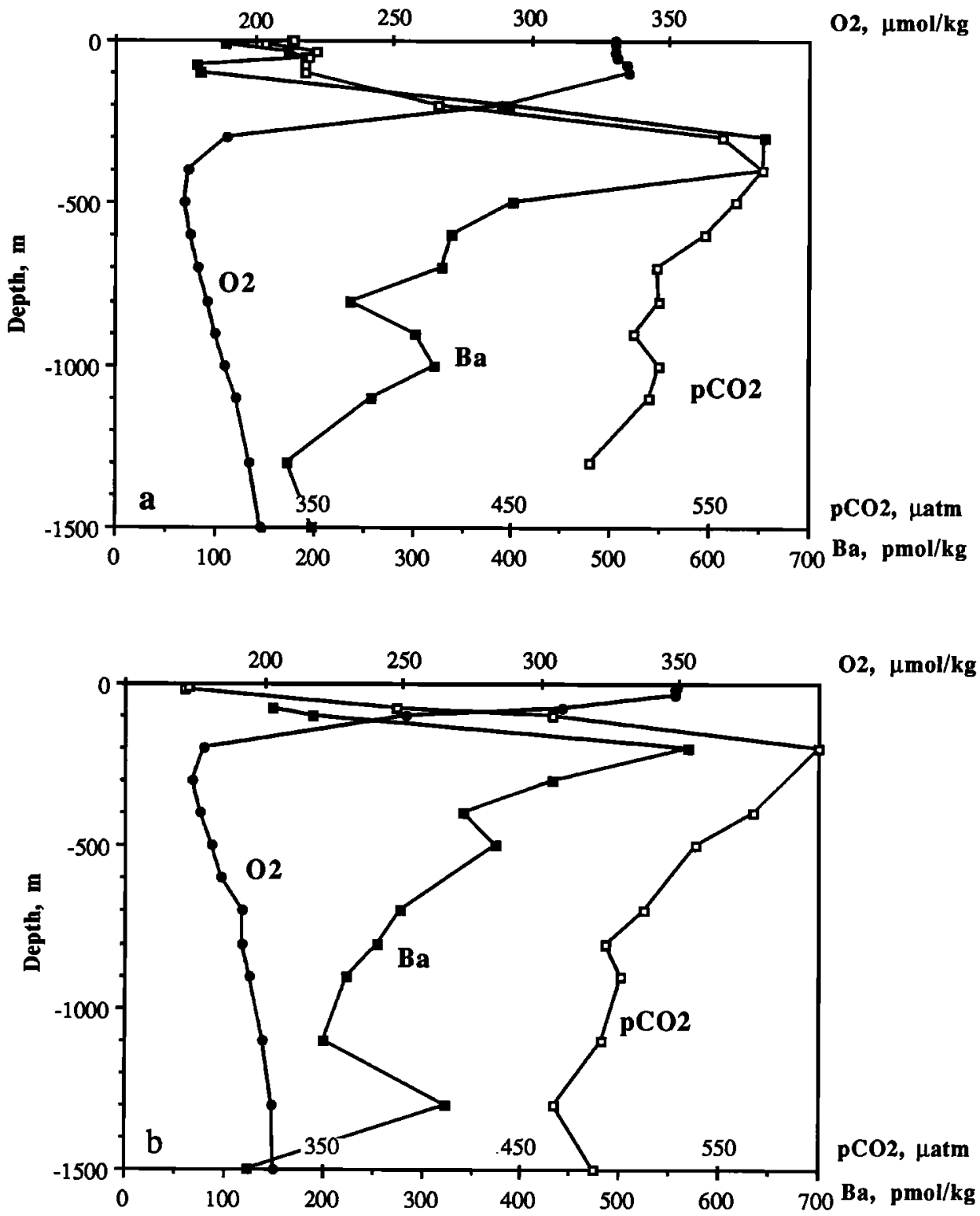

Fig. 2. Vertical profiles of particulate barium (solid squares, pmol kg-1), dissolved oxygen (solid circles, $\mu \mathrm{mol} \mathrm{kg-1}$ ) and $\mathrm{pCO}_{2}$ (open circles, $\mu \mathrm{atm}$ ) at stations (a) 75, (b) 78, (c) 87, and (d) 99 . For station 78 the surface water $\mathrm{Ba}$ maximum ( $\mathrm{Ba}>1000 \mathrm{pmol} \mathrm{kg}^{-1}$; see Table 1) is not shown.

Front. A significant correlation is apparent $(r=0.82 ; \mathrm{p}<$ 0.01 ), showing increased barium-barite content for increasing oxygen consumption.

However, this observed relationship between oxygen and barium appears to hold only for the stations south of the Polar Front. Stations 95, 98, and 99 located north of the Polar Front do not fit it, showing lower $\mathrm{Ba}$ concentrations than predicted by the $\mathrm{Ba}-\mathrm{O}_{2}$ relationship (Figure 3). For the stations located within the Antarctic Circumpolar Current, the observations suggest some causal relation between consumption of oxygen due to heterotrophic activity and production, or rather release, (as discussed below) of barite crystals. They also suggest the oxygen minimum waters to flow northnortheastward, away from the divergence. This is also emphasized by the following observations.

1. For the region south of the Polar Front the general south to north- northeast decrease of oxygen in the oxygen minimum is paralleled by an increase of nitrate in the nitrate maximum layer (generally located slightly above the oxygen minimum; nitrate data are reported by Poisson et al. [1990]), with oxygen and nitrate 

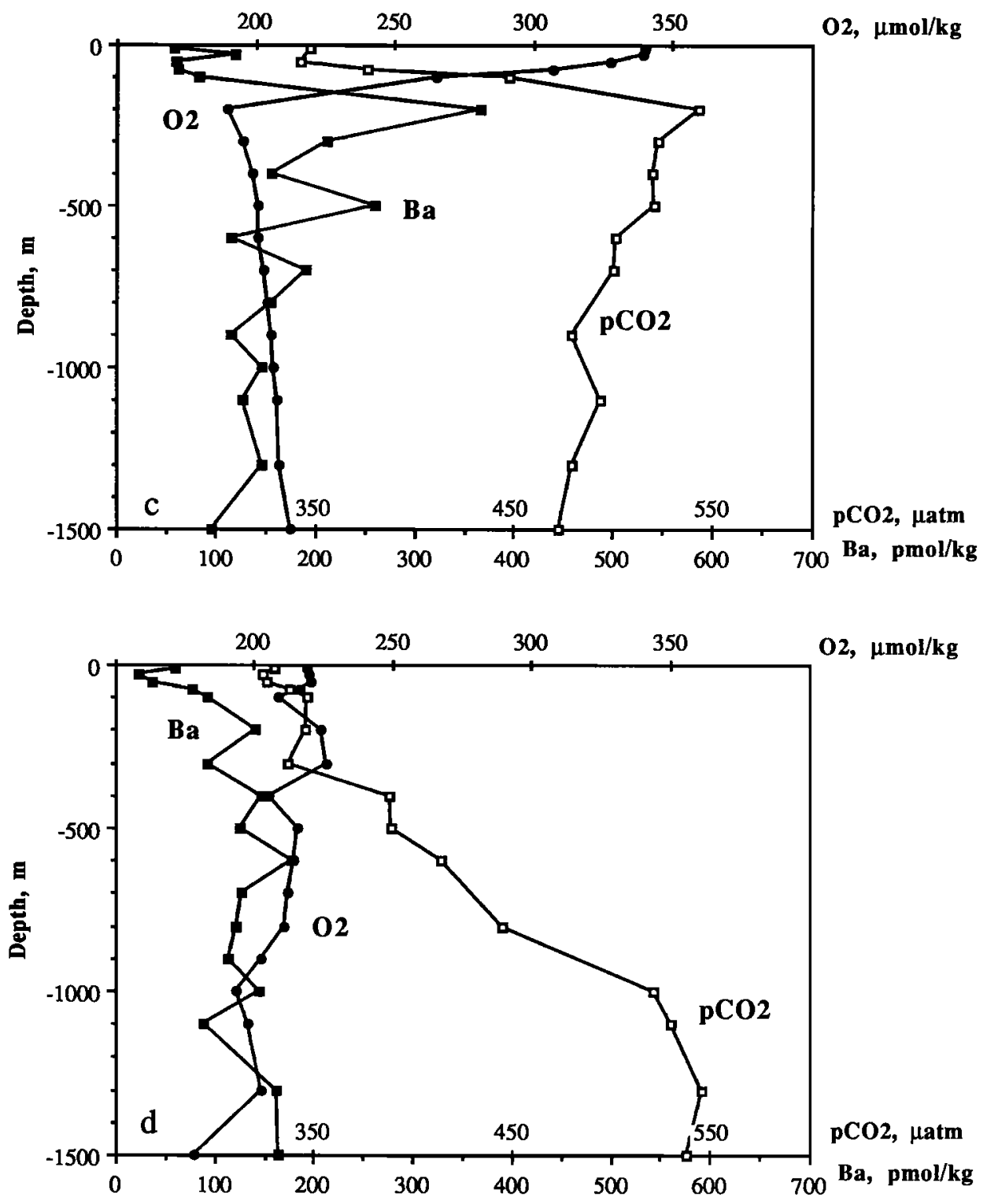

Fig. 2. (continued)

significantly correlated (Figure 4). The slope of this relationship $\left(\Delta \mathrm{O}_{2} / \Delta \mathrm{NO}_{3}\right)$ is $17.2 \mathrm{l}$, which is close to the Redfield ratio of 8.6 [Redfield et al., 1963]. For the area investigated here, we believe it is not realistic to inspect for oxygen consumption along isopycnal surfaces, due to the sharp upward bending of the isopycnals as they approach the divergence. Indeed, in such a situation the effect due to the oxidation of organic matter transported by advection along an isopycnal surface is likely to be expressed at a different depth than the effect due to the oxidation of organic matter carried by the large, vertically settling particles. We also inspected the $\mathrm{O}_{2} / \mathrm{NO}_{3}$ relationship along isopycnal surfaces $\left(\sigma_{\theta} 27.600\right.$ and 27.700) encompassing the oxygen minimum. In this case the correlations are less significant, and $\Delta \mathrm{O}_{2} / \Delta \mathrm{NO}_{3}$ ratios are $\geq|20|$. This is much in excess of values observed along isopycnal surfaces in the Atlantic, Indian, and Pacific oceans $\left(\Delta \mathrm{O}_{2} / \Delta \mathrm{NO}_{3}=|11|\right.$ [Takahashi et al., 1985; Broecker et al., 1985; Peng and Broecker, 1987]).

2. As was stated above, the Ba-oxygen relationship holds only for stations located within the Antarctic Circumpolar Current. North of the Polar Front the oxygen minimum occurs much deeper $(>1400 \mathrm{~m})$ in the water column, and values of the $\mathrm{Ba}$ maximum are smaller than expected from the described $\mathrm{Ba}_{2} \mathrm{O}_{2}$ relationship. This indicates the Ba maximum in the circumpolar current to be a local phenomenon reflecting 


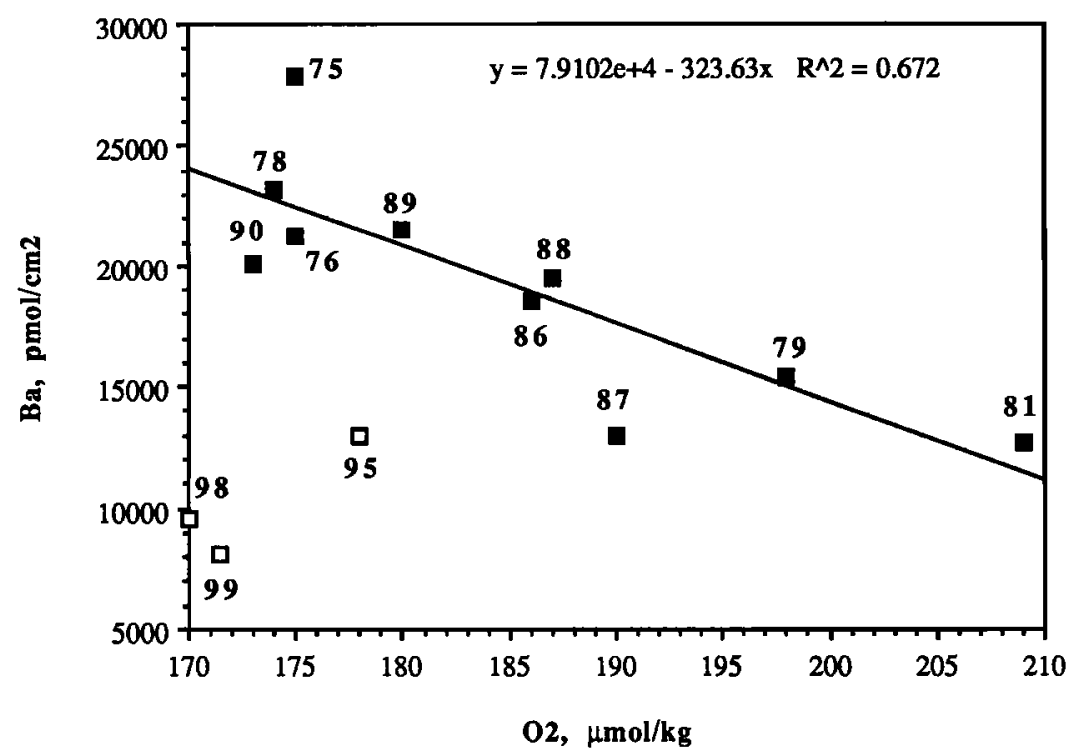

Fig. 3. Particulate barium ( $\mathrm{pmol} \mathrm{cm}-2$ ), integrated over the barium maximum versus dissolved oxygen $\left(\mu \mathrm{mol} \mathrm{kg}{ }^{-1}\right)$ in the oxygen minimum for stations south of the Polar Front.

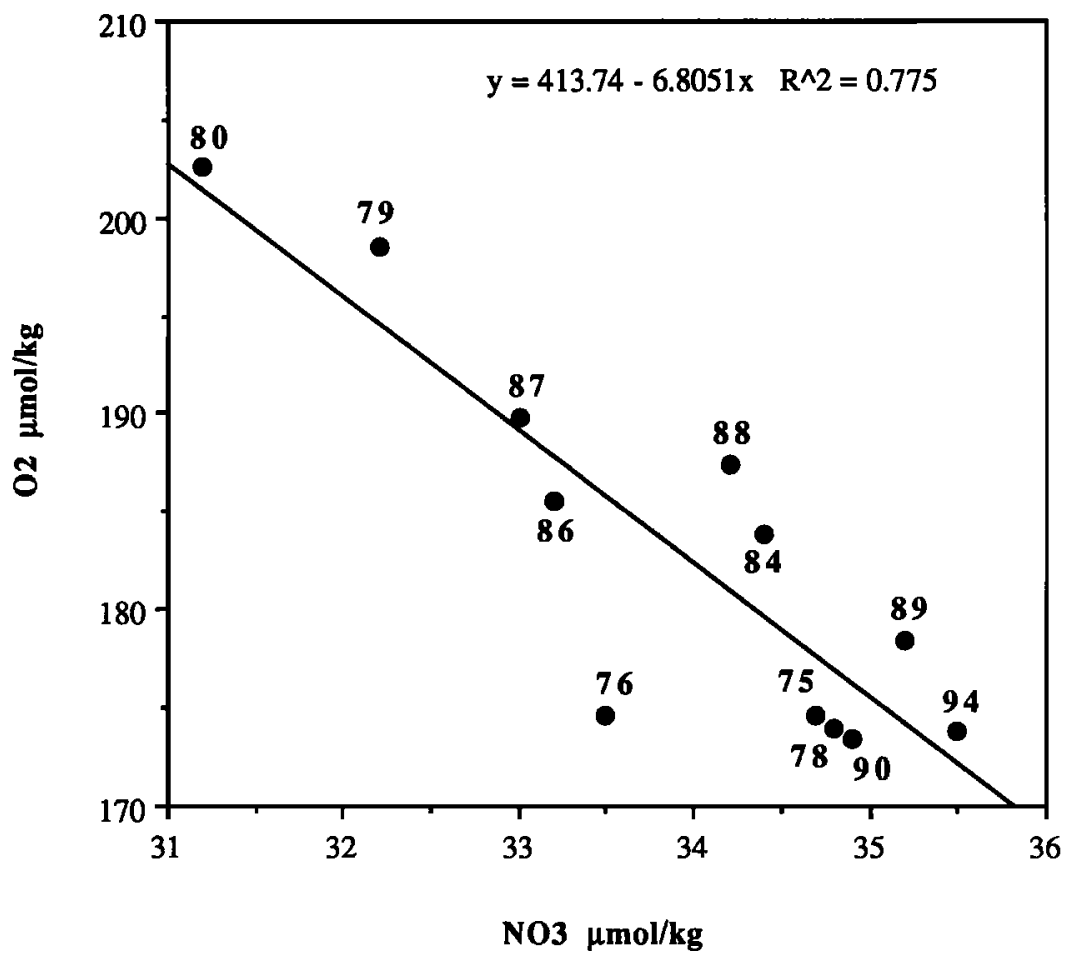

Fig. 4. Dissolved oxygen $(\mu \mathrm{mol} \mathrm{kg}-1)$ in the oxygen minimum versus nitrate $\left(\mu \mathrm{mol} \mathrm{kg}^{-1}\right)$ in the nitrate maximum for stations south of the Polar Front.

the particular properties of this water mass. Indeed, if both the Ba maximum and the oxygen minimum were properties advected from the adjacent oceanic basins towards the divergence, the $\mathrm{Ba}$ maximum north of the
Polar Front should be as high or higher than south of the front; this is not observed.

The similarity between the observed stoichiometry of nitrate and oxygen in the oxygen minimum region with 
that predicted by Redfield ratio values, and the discontinuity in the values of the barium maximum across the Polar Front suggest that water in the oxygen minimum layer (i.e. the 200-500 $\mathrm{m}$ depth layer) increases in age in a general north- northeastward direction. We believe our conclusions are also supported by Toggweiler et al.'s [1989] observations indicating that water upwelling at the divergence is largely Circumpolar Water containing relatively little deep water from the major basins to the north.

In the following we check whether the decrease of oxygen in the oxygen minimum over a given distance is compatible with known rates of primary production. Therefore we will follow a parcel of water in the oxygen minimum layer from the source region to some point located downstream in the circumpolar current. We choose station 87 as the source region since it is located upstream, close to the Antarctic Divergence. Stations 78, 76 , and 75 , located to the northeast and downstream of station 87, are choosen to represent the target region. For the circumpolar current, along the prime meridian, Whitworth and Nowlin [1987] calculated geostrophic speeds between 2 and $10 \mathrm{~cm} \mathrm{~s}^{-1}$ at $200 \mathrm{~m}$ depth. Taking a speed of $5 \mathrm{~cm} \mathrm{~s}^{-1}$ it is calculated that the distance between stations 87 and $78(2500 \mathrm{~km})$ is covered in 580 days and between stations 87 and $76,75(2000 \mathrm{~km})$ in 460 days. For a $200 \mathrm{~m}$ depth interval, centered on the oxygen minimum depth, the differences in integrated oxygen content between station 87 (the source) and stations 78,76, and 75 (the target) amount to $3400 \mathrm{mmol}$ $\mathrm{O}_{2} \mathrm{~m}^{-2}$ (87 to 78$)$ and $4100 \mathrm{mmol} \mathrm{O}_{2} \mathrm{~m}^{-2}(87$ to 76,75$)$. Taking into account the calculated transit time for the oxygen minimum water, these observed oxygen deficits reflect oxygen utilization rates of $5.9 \mathrm{mmol} \mathrm{m}^{-2} \mathrm{~d}^{-1}(87$ to 78$)$ and $8.9 \mathrm{mmol} \mathrm{m}^{-2} \mathrm{~d}^{-1}(87$ to 76,75$)$. These oxygen consumptions are equivalent to the oxidation respectively of 4.3 and $6.5 \mathrm{mmol} \mathrm{C} \mathrm{m}^{-2} \mathrm{~d}^{-1}$, using the $\Delta \mathrm{O}_{2} / \Delta \mathrm{C}$ Redfield ratio (175 / 127) from Takahashi et al. [1985] and Peng and Broecker [1987]. If we assume these rates of organic carbon oxidation to represent the totality of new production, itself representing $45 \%$ of total production in the Antarctic [Eppley and Peterson, 1979], we calculate yearly averaged daily total productions of 115 and $173 \mathrm{mg} \mathrm{C} \mathrm{m}^{-2} \mathrm{~d}^{-1}$; this is equivalent to yearly productions of 42 and $63 \mathrm{~g} \mathrm{C} \mathrm{m}^{-2}$ $\mathrm{yr}^{-1}$. These values will be compared with known rates of primary production. From the discussion on global maps of primary productivity given by Berger [1989], values for the southem ocean appear to range between 60 and $125 \mathrm{~g} \mathrm{C} \mathrm{m}^{-2} \mathrm{yr}^{-1}$. Recent seasonal primary production rates for the open southern ocean range from 100 to 350 $\mathrm{mg} \mathrm{C} \mathrm{m}^{-2} \mathrm{~d}^{-1}$ ([e.g., Wefer et al., 1982; Le Jehan and Tréguer, 1983; Lancelot et al., 1989], but occasional high values up to $2000 \mathrm{mg} \mathrm{C} \mathrm{m}^{-2} \mathrm{~d}^{-1}$ have been observed in the area of the Antarctic Peninsula [Bodungen et al., 1986]. Taking the production period to last during 4 months of the year, these seasonal productivities translate into annual productivities ranging from 12 to 43 g C m $\mathrm{Cy}^{-2}$, without considering the extreme value reported for the peninsula region. It appears that our calculated values are similar to the values deduced from global maps and to the higher values reported for seasonal primary productivity. These comparisons indicate that the observed difference in oxygen content of the oxygen minimum layer between station 87 and stations 78, 76, and 75 is consistent with the described general flow pattern of the oxygen minimum water.

It remains to be checked whether the estimated aging of the water in the oxygen minimum layer is also consistent with the observed accumulation of barite. Without considering the dissolution effect and assuming Stokes sedimentation law to apply, a 1- $\mu$ m large barite crystal will need about 5 years to cross a 200-m-thick water layer (what is about the width of the subsurface barium maximum layer). Furthermore, barite dissolution in seawater is a slow process with a dissolution rate estimated at $0.075 \mu \mathrm{m} \mathrm{yr}^{-1}$ [Dehairs, 1979; Dehairs et al., 1980]. Thus barite residence time in the oxygenminimum region can exceed the estimated aging of the water between considered source and target regions.

From the preceding discussion it appears that there is evidence for internal consistency between evolution of the oxygen minimum layer over a given distance as controlled by advection velocity and primary production on one hand, and accumulation of barite as controlled by sedimentation and dissolution on the other. Barite content thus reflects and integrates former biological processes.

\section{Origin of the Oxygen Minimum}

We propose the following scenario for the origin of the oxygen minimum, $\mathrm{pCO}_{2}$ maximum in the Antarctic Circumpolar Current. This origin is likely to be upwelling along the Antarctic Divergence. At the divergence (southernmost stations 80 and 81 ) the minimum oxygen value is $209 \mu \mathrm{M}$ at 900 to $1000 \mathrm{~m}$ depth, at $\sigma_{\theta}=27.83$. For all other stations between the divergence and the Polar Front, the 204 to $209 \mu \mathrm{M}$ oxygen range is found at the same isopycnal surface $\left(\sigma_{\theta}\right.$ between 27.83 and 27.84 ) but deeper (i.e., below the salinity maximum) than at stations 80 and 81 (Figure 5). This suggests the occurrence of the $209 \mu \mathrm{M}$ value as a minimum at stations 80 and 81 to be induced through southward advection of deep water. It seems thus likely that deep water with a low oxygen value (i.e., the 209 $\mu \mathrm{M}$ value) is originally advected to the south, rising to shallower depths along the 27.83 isopycnal surface. From the divergence onward the water carrying this oxygen value returns to the north and northeast. Its oxygen content is then affected by local processes of oxygen consumption which enhance the oxygen minimum. This scenario is also supported by the data for freon 11 and 12 [Mantisi, 1989; Poisson et al., 1990]. Freon concentrations in the oxygen minimum layer increase from the vicinity of the divergence to the north and northeast. This increasing contamination of the upwelled, freon-poor, deep water with distance from the divergence, suggests that the oxygen minimum waters are flowing away from the divergence and accumulate the freon that is penetrating from the surface layers.

\section{The Barium Maximum in the Euphotic Zone}

Barium concentrations in the euphotic layer can rise occasionally to high values. For stations 78 and 79 these maxima greatly exceed (respectively by a factor of 4 and 


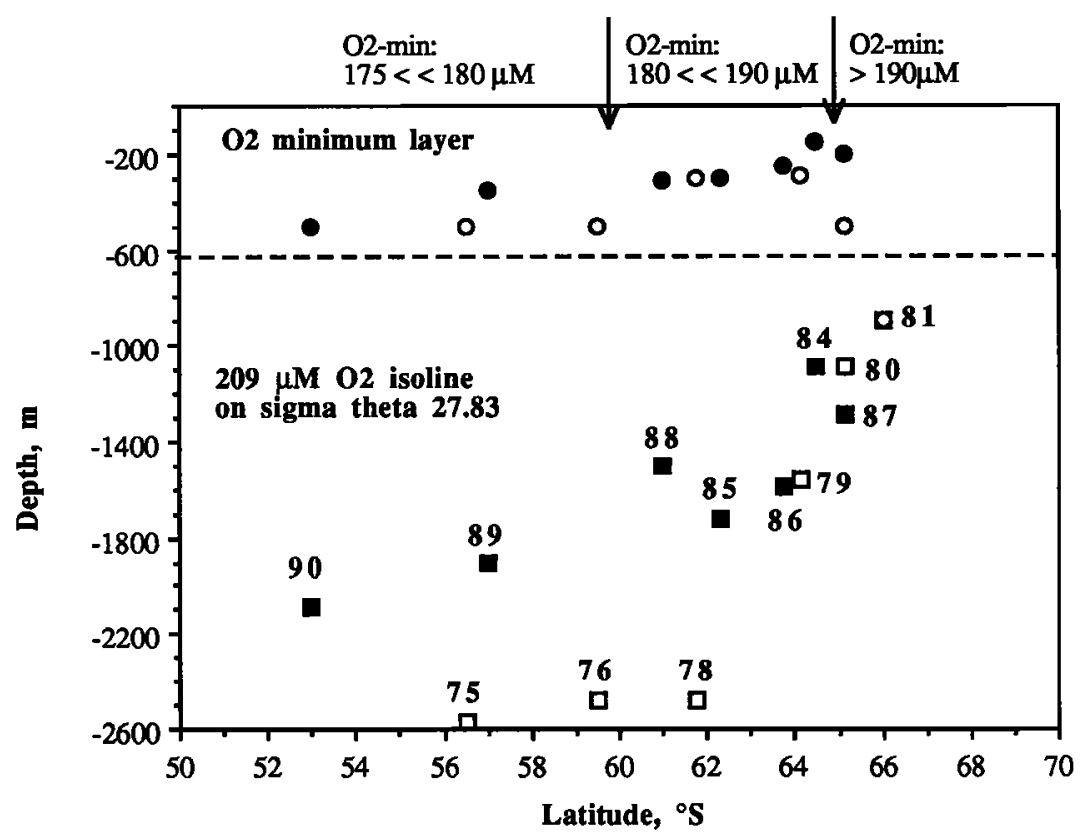

Fig. 5. Meridional section between $53^{\circ} \mathrm{S}$ and $66^{\circ} \mathrm{S}$ showing the depths at which the $209 \mu \mathrm{M}$ oxygen value (squares) and the oxygen minimum (circles) are located. Solid symbols, along $30^{\circ} \mathrm{E}$; open symbols, along $84^{\circ} \mathrm{E}$.

20)-those in the oxygen minimum layer. As shown in Figure 6, they coincide with higher chlorophyll-a concentrations (1.1 to $1.2 \mu \mathrm{g} \mathrm{L}^{-1}$ [Goffart and Hecq, $1988])$, as compared to other stations $\left(<0.5 \mu \mathrm{g} \mathrm{L}^{-1}\right)$ and the nitrate values in the surface waters are lower (station $78 ; 21.3 \mu \mathrm{mol} \mathrm{L}-1$; station $79 ; 23.5 \mu \mathrm{mol} \mathrm{L}-1$ ) than at other stations (typically 25 to $27 \mu \mathrm{mol} \mathrm{L}^{-1}$ ). For station 80 no particulate matter was sampled, but the high Chl-a

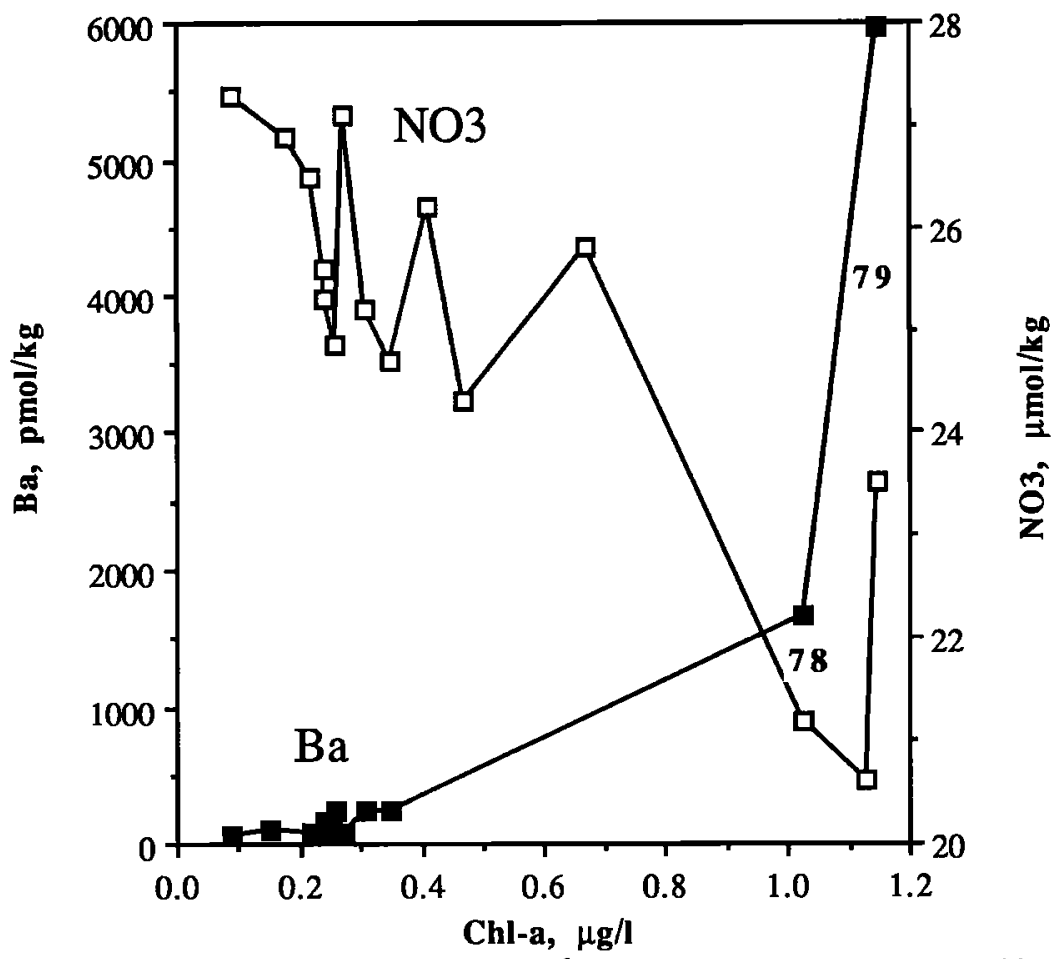

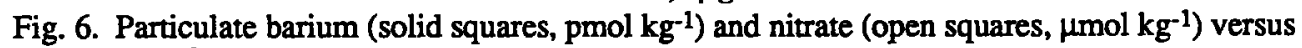
Chl- $a\left(\mu \mathrm{g} \mathrm{L}^{-1}\right)$ in the surface waters $(<50 \mathrm{~m})$. 
and the low nitrate values in the euphotic layer suggest that here also high barium concentrations were present.

This coincidence of high barium values with high chlorophyll- $a$ values suggests active production of barite. However, it is also possible that the higher phytoplankton biomass present sustains a higher biomass and activity of heterotrophs. This is suggested by the observed positive relationship between the ammonium mineralization rate and the barium content in the euphotic layer at other stations (Figure 7). Since for stations 78 and 79 no mineralization rates were measured, we do not know whether the observed relationship with barium applies also for these sites with extreme barium contents in the euphotic layer. Bacterial biomass, estimated from countings on acrydine-orange stained samples obtained during the same cruise [Dezan, 1987; Joiris et al., 1988], is not significantly higher at station $78\left(1.6 \mu \mathrm{g} \mathrm{C} \mathrm{L}^{-1}\right)$, but it is at stations 79,80 , and 81 (respectively $2.5 ; 5.1$ and $4.3 \mu \mathrm{g} \mathrm{CL}^{-1}$ ). However, for station 81 this high bacterial biomass does not coincide with a high barium content.

Finally, SEM-EMP data for the $-10 \mathrm{~m}$ sample at station 76 show barite to occur mainly as small crystals $(<0.5$ $\mu \mathrm{m}$ ) forming aggregates associated with larger particles composed of mixed biogenic debris including diatom tests. This observation is in favor of passive barite formation within microenvironments, composed here of mixed aggregates of organic detritus and biogenic debris.

Although these facts do not give clear-cut evidence, the majority of the observations is in favor of passive production of barite in the euphotic layer. They also indicate that there is a time lag between the observed phenomena in the oxygen minimum layer and the euphotic layer. Indeed, biological activity, which is responsible for the extreme barium content in the euphotic layer at station 79 (Table 1), did not yet have any impact on the barium content in the oxygen'minimum area at the time of sampling, as evidenced by the low $\mathrm{Ba}$ value in the subsurface barium maximum at this station.

\section{The Production and Redistribution of Barite}

From the SEM-EMP investigations in this study, it appears that in the surface waters barite is associated with composite biogenic aggregates, while at greater depths barite crystals occur as free particles. SEM-EMP investigations on samples we took during a more recent southern ocean cruise (EPOS-2; November-December 1988; Scotia-Weddell Confluence area), also showed occurrence of large $(>100,<1000 \mu \mathrm{m})$, loosely packed bioaggregates carrying barite crystals. Such aggregates were abundant in the first $200 \mathrm{~m}$ of the water column but virtually absent below. These observations are very much in accord with those made by Bishop [1988] showing, for surface waters in the NW and SE Atlantic Ocean, total Ba to be associated with the size fraction $>$ $53 \mu \mathrm{m}$. At 100 to $200 \mathrm{~m}$ depth the largest fraction of barium is seen to shift toward the $<53 \mu \mathrm{m}$ size class [Bishop, 1988].

These observations suggest that mixed biogenic aggregates represent the microenvironments where barite microcrystals are formed. These aggregates settle out of the mixed layer but are disintegrated just below the first

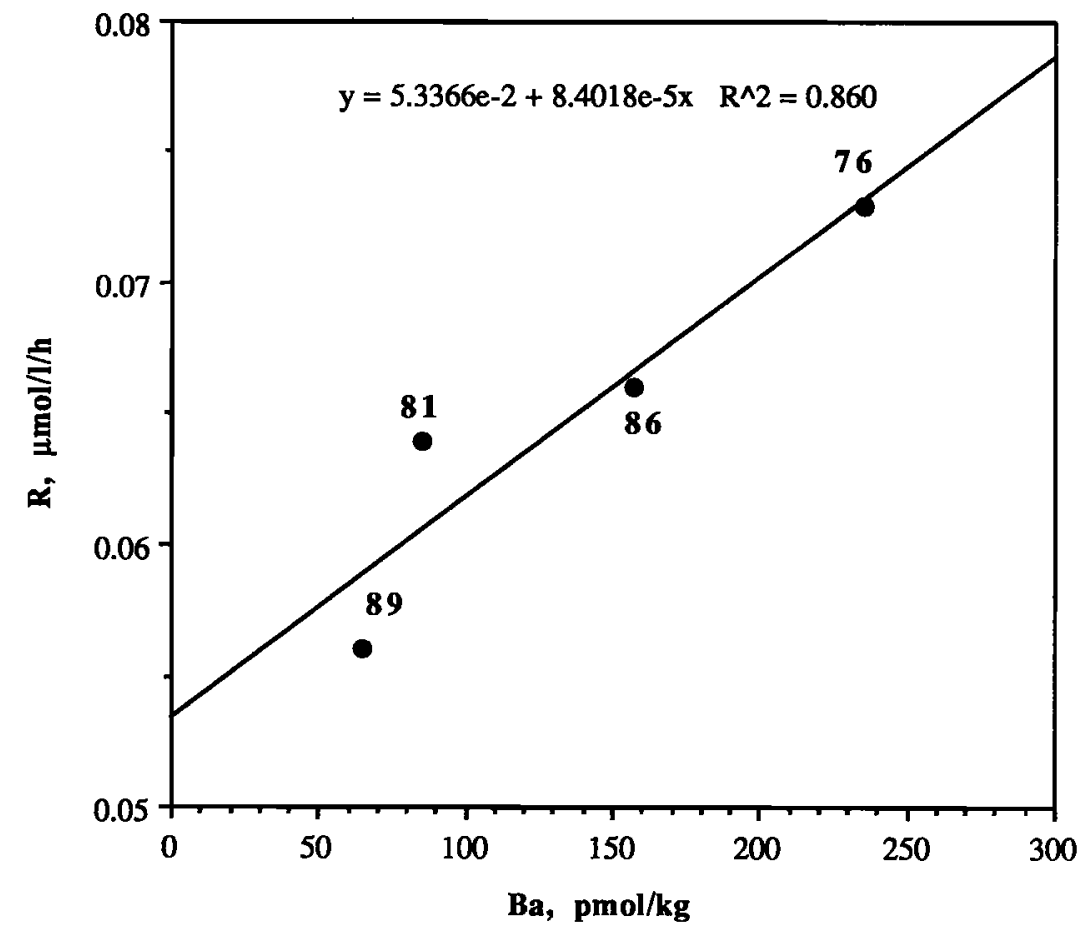

Fig. 7. Mineralization rate of ammonia $\left(\mu \mathrm{mol} \mathrm{L} \mathrm{L}^{-1} \mathrm{~h}^{-1}\right)$ versus particulate barium (pmol kg-1) content in the surface waters $(<50 \mathrm{~m})$. 
few hundred meters of depth, setting free the micronsized barite crystals they carry. These discrete barite particles will now accumulate as a result of their small settling and dissolution rates. At the $\mathrm{Ba}$ maximum depth, barite content is no longer related to instantaneous biological activity, as it probably is in the euphotic layer, but rather reflects former biological activities.

The possibility that barite forms in situ at the Ba maximum depth as a result of in situ bacterial activity seems unlikely. Indeed, instantaneous heterotrophic activity, as reflected by the measured ammonium mineralization rates, is inversely correlated with $\mathrm{Ba}$ at these depths. Here oxygen content seems to control mineralization rates as indicated by the positive correlation we observe between both parameters.

\section{CONCLUSIONS}

The distribution of particulate $\mathrm{Ba}$ in surface waters of the mixed layer is closely linked to the biological activity. At depth, the coincidence of the Ba-barite maximum with the oxygen minimum and the inverse correlation between these extremes point towards a significant, local, biological control on the oxygen minimum. The identification of biological activity, besides advection, as a main process controling the oxygen minimum has set constraints as to the flow direction of these oxygen minimum waters. These waters appear to be originally advected to the south as oxygen poor deep waters. They well up at the Antarctic Divergence from where they spread out partly to the north-northeast. From then on, oxidation of settling organic matter, produced in local surface waters, consumes oxygen and induces an oxygen minimum. The distribution of $\mathrm{pCO}_{2}$ in this region is the mirror distribution of $\mathrm{O}_{2}$ and therefore also reflects the combined effect of physical, chemical and biological processes. The situation described is probably typical for the area between the Antarctic Divergence and the Polar Front.

While the data presented here again highlight the link between barite and biological activity, which is essential in the study of the carbon cycle, they also reveal the important role of biogenic microenvironments, either as formation sites of barite, or as carriers of barite to the deeper layers, thereby lending strong support to the original Chow and Goldberg [1960] model.

Acknowledgments. We are grateful to $\mathrm{H}$. Neybergh, at the Belgian Geological Survey, for providing access to the ICP-OES equipment in his laboratory and assistance during the measurements. We thank M. Pamatmat for his comments and discussions. The crew of the Marion Dufresne as well as the TAAF administration are thanked for their logistical support. This research is part of the "Belgian Scientific Research Programme on Antarctica -Phase 1" (Prime Minister's Services, Science Policy Office). The scientific responsibility is assumed by its authors. F.D. is a research associate at the National Fund for Scientific Research, Belgium.

\section{REFERENCES}

Armstrong, F. A. I., C. R. Steins and J. D. H. Strickland, The measurement of upwelling and subsequent biological processes by means of the Technicon autoanalyzer and associated equipment, Deep Sea Res., 14, 381-389, 1967.

Arrhenius, G., and E. Bonatti, Neptunism and vulcanism in the ocean, in Progress in Oceanography, vol. 3, edited by M. Sears, pp. 7-22, Pergamon, New York, 1965.

Berger, W. H., Global maps of ocean productivity, in Productivity of the ocean: Present and Past, Dahlem Workshop Reports, Life Sci. Res. Rep. 44, edited by W.H. Berger, V.S. Smetacek, and G. Wefer, pp. 429455, John Wiley, New York, 1989.

Bishop, J. K. B., The barite-opal-organic carbon association in oceanic particulate matter, Nature, 332 , 341-343, 1988.

Bishop, J. K. B., Regional extremes in particulate matter composition and flux: Effects on the chemistry of the ocean interior, in Productivity of the ocean: Present and Past, Dahlem Workshop Reports, Life Sci. Res. Rep. 44, edited by W.H. Berger, V.S. Smetacek and G. Wefer, pp. 117-137, John Wiley, New York, 1989.

Bishop, J. K. B., and J. M. Edmond, A new large volume filtration system for the sampling of oceanic particulate matter, J. Mar. Res., 34, 181-198, 1976.

Bodungen, B. v., V. S. Smetacek, M. M. Tilzer, and B. Zeitschel, Primary production and sedimentation during spring in the Antarctic Peninsula region, Deep Sea Res., 33, 177-194, 1986.

Broecker, W. S., T. Takahashi, and T. T. Takahashi, Sources and flow pattern of deep-ocean waters as deduced from potential temperature, salinity and initial phosphate concentrations, J. Geophys. Res., 90, 6925-6939, 1985.

Brook, A., A. Fotheringham, J. Bradley, and A. Jenkins, Barium accumulation by desmids of the genus Closterium (Zygnemaphyceae), Br. Phycol. J., 15, 261-264, 1980.

Burman, J.-O., C. Pontér, and K. Boström, Metaborate digestion procedure for inductively coupled nlasma-optical emission spectrometry, An. Chem., 50, 679 . 680, 1978.

Chow, T. J., and E. D. Goldberg, On the marine geochemistry of barium, Geochim. Cosmochim. Acta, 20, 192 - 198, 1960.

Church, T. M., Marine barite, Ph.D. thesis, 100 pp., Univ. of Calif., San Diego, 1970.

Church, T. M., Marine barite, in Reviews in Mineralogy, vol. 6, edited by R.G. Burns, pp. 175209, Mineralogical Society of America, Washington, D.C., 1976.

Church, T. M., and K. Wolgemuth, Marine barite saturation, Earth Planet. Sci. Lett., 15, 35-44, 1972.

Dehairs, F., Discrete suspended particles of barite and the barium cycle in the open ocean, Doctoral thesis, 285 pp., Vrije Univ.Brussel, Brussels, 1979.

Dehairs F., and L. Goeyens, The biogeochemistry of barium in the southern ocean, in Belgian Scientific Research Programme on Antarctica: Scientific Results of Phase One (Oct. '85-Jan. '89), vol. II, part A, Marine Geochemistry, edited by S. Caschetto, pp. 1100 , Prime Minister's Services, Science Policy Office, Brussels, 1989.

Dehairs, F., R. Chesselet, and J. Jedwab, Discrete suspended particles of barite and the barium cycle in the 
open ocean, Earth Planet. Sci. Lett., 49, 528-550, 1980.

Dezan, Y., Bacterial biomass and activity in North Sea and Antarctic waters, M. Sc. thesis, 111 pp., Vrije Univ. Brussel, Brussels, 1987.

Dymond, J., Particulate barium fluxes in the oceans: An indicator of new productivity (abstract), Eos. Trans. $A G U, 56,1275,1986$.

Eppley, R. W., and B. J. Peterson, Particulate organic matter flux and planktonic new production in the deep ocean, Nature, 282, 677-680, 1979.

Finlay, B. J., N. B. Hetherington, and W. Davison, Active biological participation in lacustrine barium chemistry, Geochim. Cosmochim. Acta, 47, 13251329, 1983.

Gayral, P., and J. Fresnel, Exanthemachrysis gayraliae Lepailleur (Prymnesiophyceae, Pavlovales): Ultrastructure et discussion taxinomique, Protistologica, 1, 271-282, 1979.

Goeyens, L., and F. Dehairs, Study of the remineralization process by $\mathrm{N}-15$ isotope dilution experiments, in Les Rapports des Campagnes d la Mer, MD53 I INDIGO3 , a bord du "Marion Dufresne", 3 janvier-27 fevrier 1987, Publ. 87-01, Fascicule 3, edited by A. Poisson and S. Caschetto, pp. 5-21, Les Publications de la Mission de Recherche des Terres Australes et Antarctiques Françaises, Paris, France, 1989.

Goffart, A., and J. -H. Hecq, Distribution of phytoplanktonic parameters in the Indian sector of the southern ocean during INDIGO 3 cruise, in Antarctica, Proceedings of the Belgian National Colloquium on Antarctic Research, Brussels October 20, 1987, pp. 47-166, Prime Minister's Services, Science Policy Office, Brussels, 1988.

Goldberg, E., and G. Arthenius, Chemistry of pelagic sediments, Geochim. Cosmochim. Acta, 13, 153 $212,1958$.

Grasshoff, K., A multiple channel system for nutrient analysis in seawater with analog and digital data record, in Advances in Automated Analysis, Technicon International Symposium, Chicago, pp. 133-145, 1969.

Hanor, J. S., Rates of barium accumulation in the Equatorial Pacific (abstract), Geol. Soc. Am. Abstr. Programs, 4, 526, 1972.

Harrison, W. G., Use of isotopes, in Nitrogen in the Marine Environment, edited by E.J. Carpenter and D.G. Capone, pp. 763-807, Academic, San Diego, Calif., 1983.

Hubert, G., N. Rieder, G. Schmitt, and W. Send, Accumulation of barium in Müller's bodies of the Loxodidae (Ciliata, Holotricha), ZNaturforsch., C. 30, 422-423, 1975.

Joiris, C., W. Overloop, M. Frankignoulle, and J. M. Bouquegneau, Preliminary discusion of the results obtained in Antarctica during the austral summer 19861987: Plankton ecology and ecotoxicology, in Antarctica, Proceedings of the Belgian National Colloquium on Antarctic Research, Brussels October 20, 1987, pp. 97-113, Prime Minister's Services, Science Policy Office, Brussels, 1988.

Lancelot, C., G. Billen, and S. Mathot, Ecophysiology of phyto- and bacterioplankton growth in the southern ocean, in Antarctica, Belgian Scientific Research Programme on Antarctica: Scientific Results of Phase One (Oct. '85 - Jan. '89), vol. I, Plankton Ecology, edited by S. Caschetto, pp. 4-97, Prime Minister's Services, Science Policy Office, Brussels, 1989. Le Jehan, S., and P. Tréguer, The distribution of inorganic nitrogen, phosphorus, silicon and dissolved organic matter in surface and deep waters of the southem ocean, in Antarctic Nutrient Cycles and Food Webs, edited by W.R. Siegfrid et al., pp. 22-29, Springer-Verlag, New York, 1983.

Lutjeharms, J. R. E., N. H. Walters, and B. R. Allanson, Oceanic frontal systems and biological enhancement, in Antarctic Nutrient Cycles and Food Webs, .edited by W.R. Siegfrid et al., pp. 11-21, Springer-Verlag, New York, 1983.

Mantisi, F., Utilisation des fréons 11 et 12 comme traceurs des masses d'eaux océaniques dans l'océan Indien sud ouest, Perspectives quant à la pénétration du $\mathrm{CO}_{2}$ anthropique, Thèse de Doctorat, $178 \mathrm{pp}$., Univ.Paris 6, Paris, 1989.

Peng, T. -H., and W. S. Broecker, C/ P ratios in marine detritus, Global Biogeochem. Cycles, 1, 155161, 1987.

Poisson, A., B. Schauer, and C. Brunet, Les Rapports des campagnes a la Mer, MD 53 / INDIGO 3 d bord du "Marion Dufresne", 3 janvier -27 février 1987, Publ. 87-01, Fascicule 2, 269 pp., Les Publications de la Mission de Recherche des Terres Australes et Antarctiques Françaises, Paris, 1990.

Raeymakers, B., Characterization of particles by automated electron probe microanalysis, Doctoral thesis, 295 pp., Univ. of Antwerp, Antwerp, Belgium 1986.

Redfield, A. C., B. H. Ketchum, and F. A. Richards, The influence of organisms on the composition of seawater, in The Sea, vol. 2, edited by M.N. Hill, pp. 26-77, Interscience, New York, 1963.

Rhein M., and R. Schlitzer, Radium-226 and barium sources in the deep east Atlantic, Deep Sea Res., 35, 1499-1510, 1989.

Schmitz, B., Barium, equatorial high productivity, and the northward wandering of the Indian continent, Paleoceanography, 2, 63-77, 1987.

Storms, H., Quantification of automated electron microprobe $\mathrm{X}$-ray analysis and application in aerosol research, Doctoral thesis, 270 pp., Univ. of Antwerp, Antwerp, Belgium, 1988.

Takahashi, T., W. S. Broecker, and S. Langer, Redfield ratio based on chemical data from isopycnal surfaces, J. Geophys. Res., 90, 6907-6924, 1985.

Toggweiler, J. R., K. Dixon, and K. Bryan, Simulations of radiocarbon in a coarse-resolution world model, 1, Steady state prebomb distributions, $J$. Geophys. Res., 94, 8217-8242, 1989.

Wefer, G., E. Suess, W. Balzer, G. Liebezeit, P. J. Müller, C. A. Ungerer, and W. Zenk, Fluxes of biogenic components from sediment trap deployment in circumpolar waters of the Drake Passage, Nature, 299, 145-147, 1982.

Whithworth, T., III, and W. D. Nowlin, Jr., Water masses and currents of the southern ocean at the Greenwich meridian, J. Geophys. Res., 92, 64626476, 1987. 
P. Bernard, Departement Scheikunde, Universitaire Instelling Antwerpen, Universiteitsplein 1, B-2610 Wilrijk, Belgium.

R. Chesselet, CNRS-INSU, 15, Quai Anatole France, 75700 Paris, France.

F. Dehairs, L. Goeyens, and N. Stroobants, ANCH Vrije Universiteit Brussel, Pleinlaan 2, B-1050 Brussels, Belgium.
C. Goyet and A. Poisson, LPCM, Université Pierre et Marie Curie, Tour 24-25, 4 Place Jussieu, 75230 Paris, France.

(Received February 15, 1990;

revised June 14, 1990;

accepted July 17, 1990.) 